\title{
Cluster Wild Bootstrapping to Handle Dependent Effect Sizes in Meta-Analysis with a Small Number of Studies
}

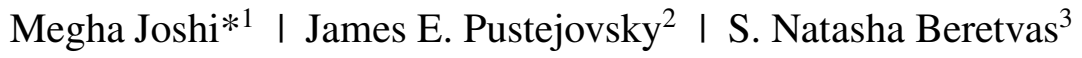

\author{
${ }^{1}$ American Institutes for Research \\ ${ }^{2}$ The University of Wisconsin at Madison \\ ${ }^{3}$ The University of Texas at Austin \\ Correspondence \\ * Megha Joshi, Corresponding address. \\ Email: megha.j456@gmail.com
}

\section{Present Address}

4700 Mueller Blvd, Austin, TX 78723

Forthcoming in Research Synthesis Methods. This paper is not the version of record and may not exactly replicate the final, published version of the article. The final article will be available, upon publication, via its DOI:

10.1002/jrsm. 1554

\begin{abstract}
Summary
The most common and well-known meta-regression models work under the assumption that there is only one effect size estimate per study and that the estimates are independent. However, meta-analytic reviews of social science research often include multiple effect size estimates per primary study, leading to dependence in the estimates. Some meta-analyses also include multiple studies conducted by the same lab or investigator, creating another potential source of dependence. An increasingly popular method to handle dependence is robust variance estimation (RVE), but this method can result in inflated Type I error rates when the number of studies is small. Small-sample correction methods for RVE have been shown to control Type I error rates adequately but may be overly conservative, especially for tests of multiplecontrast hypotheses. We evaluated an alternative method for handling dependence, cluster wild bootstrapping, which has been examined in the econometrics literature but not in the context of meta-analysis. Results from two simulation studies indicate that cluster wild bootstrapping maintains adequate Type I error rates and provides more power than extant small-sample correction methods, particularly for multiplecontrast hypothesis tests. We recommend using cluster wild bootstrapping to conduct hypothesis tests for meta-analyses with a small number of studies. We have also created an R package that implements such tests.
\end{abstract}

\section{KEYWORDS:}

cluster wild bootstrap; meta-analysis; dependence; robust variance estimation

\section{1 | INTRODUCTION}

Typical methods to conduct meta-analysis—-pooling effect sizes or analyzing moderating effects with meta-regression-work under the assumption that the effect size estimates are independent. However, primary studies often report multiple, statistically dependent estimates of effect sizes. Dependence can occur through two broad structures: correlated effects and hierarchical effects. Correlated effects occur when primary studies collect multiple, correlated measures of an outcome, collect repeated measures of the outcome(s), or compare multiple treatment groups to the same control group. ${ }^{1,2}$ Hierarchical effects can occur when primary meta-analytic studies include multiple experiments conducted by the same laboratory or investigator, creating dependence in the effect size parameters. ${ }^{2}$

Researchers may be inclined to ignore dependence and use methods that assume that each effect size estimate is independent. However, doing so can result in inaccurate standard errors and, consequently, hypothesis tests with incorrect Type I 
error rates and confidence intervals with incorrect coverage levels. ${ }^{1}$ Other, ad hoc methods for handling dependent effect sizes include averaging effect sizes by study, selecting a single estimate per study, or running multiple meta-analytic models with different subsets of the data. These methods result in loss of information, and are not suitable for studying within-study variation in effect sizes or differential effects across subsets of effect sizes. ${ }^{2}$

A more principled solution for handling dependence would be to use a multivariate model, which explicitly models the structure of dependence among effect size estimates. ${ }^{1,2,3,4}$ Standard methods for estimating such multivariate models require knowledge of the correlations or covariances between pairs of effect size estimates within each study. Formulas for calculating such correlations are available, ${ }^{5}$ but they require summary statistics which primary studies often fail to report. Thus, standard multivariate models are often infeasible in practice. ${ }^{\dagger}$

\section{1 | Robust Variance Estimation}

Hedges and colleagues ${ }^{2}$ proposed another procedure to handle dependence, robust variance estimation (RVE), that provides a means of including all available effect size estimates (i.e., without aggregating or sub-setting the data), while also remaining feasible in practice. RVE does not require the exact covariance structure between the effect size estimates. Instead, it uses a working model that entails making rough, approximate assumptions about the dependence structure. To allow for the possibility that the working model is mis-specified, the approach uses robust, sandwich-type estimators to obtain standard errors, hypothesis tests, and confidence intervals for average effect sizes and meta-regression coefficients. ${ }^{2,9}$ Because it allows for flexible modeling of dependent effect sizes without requiring detailed empirical information about the correlations between effect size estimates, RVE has become a widely used tool for meta-analyses in the social sciences.

As originally developed by Hedges and colleagues, ${ }^{2}$ the sandwich estimators used in RVE rely on asymptotic approximations, the accuracy of which requires a large number of independent studies. If the number of studies in a meta-analysis is small, RVE can result in downwardly biased standard errors and inflation of Type 1 error rates. ${ }^{2,9}$ Hedges and colleagues ${ }^{2}$ suggested, as a rough guideline, that a minimum of 40 studies might be needed to trust large-sample approximations. However, subsequent work ${ }^{9,10}$ has shown that the adequacy of the large-sample approximations depends on factors beyond just the total number of studies, making it difficult to provide any simple rule of thumb about what counts as large.

Tipton ${ }^{9}$ and Tipton and Pustejovsky ${ }^{10}$ have introduced small-sample corrections for RVE for tests of single coefficients and for multiple-contrast hypotheses, respectively. Tipton ${ }^{9}$ showed, through simulations, that the CR2 adjustment method with the Satterthwaite degrees of freedom resulted in close-to-nominal Type I error rates. Moreover, Tipton ${ }^{9}$ showed that smallsample size itself was not the only important factor that could influence the performance of RVE. The distribution of the covariates-for example, imbalanced categories or outliers in covariates-can also influence the performance of RVE. For multiple-contrast hypothesis tests, Tipton and Pustejovsky ${ }^{10}$ recommended a method (HTZ), which is an extension of the CR2 correction method with the Satterthwaite degrees of freedom. Compared to several alternative small-sample corrections, the HTZ test resulted in Type 1 error rates closest to the nominal rate of 0.05 . However, HTZ had below-nominal Type 1 error rates for tests of multiple-contrast hypotheses-particularly tests involving a larger number of contrasts. This suggests that the HTZ test may have sub-optimal power for multiple-contrast tests. In this study, we consider bootstrapping methods that may yield higher power while still retaining correctly calibrated Type 1 error rates in small samples.

\section{2 | Bootstrapping}

Bootstrapping is a technique to estimate unknown quantities such as standard errors, confidence intervals, and p-values from statistical models. The general idea behind bootstrapping is to emulate the unknown sampling distribution of an estimator or test statistic by re-sampling many times from the original dataset— that is, re-sampling from the sample distribution in order to approximate the process of sampling from the population distribution. ${ }^{11}$

Many different variants of bootstrapping have been proposed, with different assumptions and different properties. ${ }^{12}$ Perhaps the most common bootstrapping technique is called the pairs bootstrap, which involves re-sampling with replacement

\footnotetext{
${ }^{\dagger}$ Recent methodological work ${ }^{6,7,8}$ has sought to develop alternative estimation methods for multivariate meta-analysis that do not require knowledge of the correlations among outcomes. Currently, these methods are limited to meta-analysis of strictly bivariate ${ }^{6,8}$ or multivariate ${ }^{7}$ effect sizes, meaning meta-analytic data where each study contributes at most a single effect size estimate to each of two or more classes of related outcomes, and where interest is in the distribution of effect sizes for each class of outcomes. The methods involve sandwich estimation techniques that are very similar to those used in the robust variance estimation framework.
} 
both the outcome and the predictors as a pair. ${ }^{13}$ When using clustered data, full clusters of outcomes and predictors are resampled. ${ }^{14}$ A disadvantage of this procedure is that it involves re-sampling the predictors instead of holding them constant, which can lead to poor performance when the sample size is small. ${ }^{12 \dagger}$

Another common bootstrapping technique, residual bootstrapping, involves re-sampling with replacement from the regression model residuals, then using the re-sampled residuals to calculate new outcome values for each bootstrap replication. ${ }^{12}$ When applied to clustered data, the full vectors of residuals from each cluster are re-sampled with replacement. ${ }^{14}$ Although this technique has the advantage of holding the distribution of predictors constant, it also imposes the assumption that the errors are independently and identically distributed and hence, homoskedastic. ${ }^{14}$ Further, residual bootstrapping also requires that clusters of observations do not vary in size.

A third, promising technique is known as cluster wild bootstrapping, which involves sampling transformed residuals. ${ }^{14}$ The residuals, based on a null model, are multiplied by randomly assigned weights that are constant within each cluster. ${ }^{14}$ These transformed residuals are used to generate a new outcome variable, which is then used to calculate a test statistic for each bootstrap replication. ${ }^{14}$ Like residual bootstrapping, cluster wild boostrapping has the advantage of holding the distribution of predictors constant. Unlike residual bootstrapping, cluster wild bootstrapping does not impose assumptions that the regression error vectors are identically and independently distributed nor that the clusters are all of equal size. ${ }^{14}$ In the econometrics literature, cluster wild boostrapping has been shown to adequately control Type I error rates in regression analyses of clustered data with few clusters. ${ }^{14,15}$

\section{3 | The Present Study}

Cluster wild bootstrapping is potentially a promising alternative to small-sample corrections proposed by Tipton ${ }^{9}$ and Tipton and Pustejovsky. ${ }^{10}$ Moreover, it has been used in a handful of applied meta-analytic studies as a means to handle dependent effect sizes. ${ }^{16,17,18,19}$ Although cluster wild bootstrapping is already being applied in meta-analyses, its performance has not been evaluated in the context of meta-analytic models. The purpose of the present study was, therefore, to evaluate cluster wild bootstrapping as a technique for conducting hypothesis tests in meta-regression models. Specifically, we examined whether cluster wild bootstrapping maintained Type I error rate control across multiple simulated data-generating processes. Past methodological work on small-sample corrections focused on Type I error rates but only assessed power for single-contrast t-tests. A unique contribution of the present study is that we compared the power of tests-including tests of both single- and multiple-contrast hypotheses - based on cluster wild bootstrapping to the power of currently available small-sample tests in the RVE framework.

The remainder of the paper is organized as follows. In the next section, we review the theoretical development of RVE, available small-sample corrections, and the alternative technique of cluster wild bootstrapping. We then report a small empirical application demonstrating differences among small-sample corrections and bootstrap-based tests. In subsequent sections, we detail the design and report the results of an extensive set of simulation studies. In the final section, we consider limitations, directions for further research, and implications for meta-analytic practice, and we briefly describe implementation of cluster wild bootstrap tests in the $\mathrm{R}$ package wildmeta. ${ }^{20}$

\section{2 | ROBUST-VARIANCE ESTIMATION AND CLUSTER WILD BOOTSTRAPPING}

\section{1 | Meta-regression Model}

We will review RVE methods for obtaining standard errors and conducting hypothesis tests in the framework of a meta-regression model. Consider a collection of $m$ studies, where study $j$ contributes a total of $k_{j}$ effect size estimates, for $j=1, \ldots, m$. Let $\mathbf{T}_{j}$ denote a $k_{j} \times 1$ vector of effect size estimates from study $j$. Along with effect size estimates, we have available a set of covariatesthat is, variables encoding information about study design, participant sample characteristics, intervention and comparison features, and outcome properties. Let $\mathbf{X}_{j}$ denote the $k_{j} \times p$ matrix of covariates associated with study $j$ and $\mathbf{X}=\left(\mathbf{X}_{1}^{\prime}, \mathbf{X}_{2}^{\prime}, \ldots, \mathbf{X}_{J}^{\prime}\right)^{\prime}$ denote the full design matrix. We assume throughout that $\mathbf{X}$ has full column rank, although the cluster-specific design matrices, $\mathbf{X}_{j}$, need not have full column rank. A meta-regression relates the average effect sizes to a linear combination of the covariates,

${ }^{\dagger}$ When the sample size is small, situations can arise where a certain predictor has zero variance after re-sampling, especially when whole clusters of covariates are re-sampled. This makes estimation of the regression coefficient and standard error infeasible for that re-sample. ${ }^{14}$ 
as in

$$
\mathbf{T}_{j}=\mathbf{X}_{j} \boldsymbol{\beta}+\boldsymbol{\epsilon}_{j} .
$$

In this model, $\boldsymbol{\beta}$ is a $p \times 1$ vector of meta-regression coefficients - the parameters of primary interest-and $\boldsymbol{\epsilon}_{j}$ is a $k_{j} \times 1$ vector of mean-zero errors, or deviations between the effect size estimates and their expectations based on the meta-regression model. These errors consist of estimation error as well as, potentially, heterogeneity in the true effect size parameters beyond that explained by the meta-regression model. Let $\boldsymbol{\Psi}_{j}=\operatorname{Var}\left(\boldsymbol{\epsilon}_{j}\right)$ denote the $k_{j} \times k_{j}$ variance-covariance matrix of the errors for study $j, j=1, \ldots, m$. Generally, the structure of $\boldsymbol{\Psi}_{j}$ will not be fully known.

\section{2 | Robust Variance Estimation}

In the RVE framework, the meta-regression coefficients are estimated using weighted least squares. Let $\mathbf{W}_{1}, \ldots . ., \mathbf{W}_{m}$ denote a

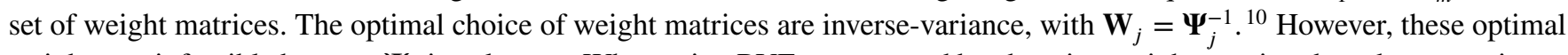
weights are infeasible because $\boldsymbol{\Psi}_{j}$ is unknown. When using RVE, we proceed by choosing weight matrices based on an estimate of an approximate working model for $\boldsymbol{\Psi}_{j}$, which need not be exactly correct. ${ }^{\dagger}$ Given a set of weights, the weighted least squares estimator of $\boldsymbol{\beta}$ can be calculated as:

$$
\mathbf{b}=\mathbf{M}\left(\sum_{j=1}^{m} \mathbf{X}_{j}^{\prime} \mathbf{W}_{j} \mathbf{T}_{j}\right), \quad \text { where } \quad \mathbf{M}=\left(\sum_{j=1}^{m} \mathbf{X}_{j}^{\prime} \mathbf{W}_{j} \mathbf{X}_{j}\right)^{-1} .
$$

For a fixed set of weights, the variance of the WLS estimator $\mathbf{b}$ is

$$
\operatorname{Var}(\mathbf{b})=\mathbf{M}\left[\sum_{j=1}^{m} \mathbf{X}_{j}^{\prime} \mathbf{W}_{j} \boldsymbol{\Psi}_{j} \mathbf{W}_{j} \mathbf{X}_{j}\right] \mathbf{M}
$$

If $\boldsymbol{\Psi}_{j}$ were fully known, then Equation 3 could be computed directly. However, the covariance structure between effect size estimates can rarely be calculated from information reported in primary studies, ${ }^{2,10}$ and so the exact structure of $\boldsymbol{\Psi}_{j}$ is not typically available.

The RVE framework uses a sandwich estimator for the variance of $\mathbf{b}$ that does not require specification of $\boldsymbol{\Psi}_{j}$. The sandwich estimator has the form

$$
\mathbf{V}^{\mathbf{R}}=\mathbf{M}\left[\sum_{j=1}^{m} \mathbf{X}_{j}^{\prime} \mathbf{W}_{j} \mathbf{A}_{j} \mathbf{e}_{j} \mathbf{e}_{j}^{\prime} \mathbf{A}_{j} \mathbf{W}_{j} \mathbf{X}_{j}\right] \mathbf{M}
$$

where $\mathbf{e}_{j}=\mathbf{T}_{j}-\mathbf{X}_{j} \mathbf{b}$ is the vector of residuals from study $j$ and $\mathbf{A}_{j}$ denotes a $k_{j} \times k_{j}$ adjustment matrix. ${ }^{9}$ In the initial formulation of RVE, ${ }^{2}$ which we shall refer to as CR0, the adjustment matrices are omitted (i.e., $\mathbf{A}_{j}^{C R 0}=\mathbf{I}_{j}$ ). With the CR0-type RVE, the true covariance matrices are estimated by the cross-product of residuals, $\mathbf{e}_{j} \mathbf{e}_{j}^{\prime}$. Although each $\mathbf{e}_{j} \mathbf{e}_{j}^{\prime}$ is a poor estimate of $\Psi_{j}$, the cross-products work sufficiently well in the aggregate, and because of the weak law of large numbers, $\mathbf{V}^{\mathbf{R}}$ converges to $\operatorname{Var}(\mathbf{b})$ as the number of studies increases. ${ }^{2,9}$

Because it relies on asymptotic approximations, the original CR0-type RVE requires a large number of studies to provide accurate standard errors. Hedges and colleagues ${ }^{2}$ suggested that CR0-type RVE might require a minimum of 40 or more studies to perform adequately. Simulation studies have shown that if the total number of studies is small (or if the total number of studies is large, but the covariates are imbalanced or have high leverage points), then using CR0 can result in downwardly biased standard errors, leading to inflation of Type I error rates for tests of single coefficients as well as for tests of multiple-contrast hypotheses. ${ }^{2,9,10}$ Hedges and colleagues, ${ }^{2}$ Tipton, ${ }^{9}$ and Tipton and Pustejovsky ${ }^{10}$ proposed several other adjustment matrices for the calculation of RVE, which we explain subsequently.

\subsection{1 | Hypothesis Tests for Single Coefficients}

Consider a hypothesis test for a single meta-regression coefficient, with null hypothesis $\mathrm{H}_{0}: \beta_{s}=0$, where $\beta_{s}$ is an element of $\boldsymbol{\beta}$. Let $b_{s}$ denote the corresponding term in $\mathbf{b}$ and $V_{s s}^{R}$ denote the $s^{\text {th }}$ diagonal element of $\mathbf{V}^{\mathbf{R}}$. A Wald test of $H_{0}$ is based on the

\footnotetext{
${ }^{\dagger}$ Hedges and colleagues ${ }^{2}$ suggested two working models, the correlated effects and hierarchical effects working models, with simplified assumptions about the dependence structure of each study. They used these working models to derive weight matrices that are not exactly inverse-variance, but aim to retain a high level of efficiency. Pustejovsky and Tipton ${ }^{21}$ proposed additional, more flexible working models that aim to better approximate the structure of the errors in the meta-regression model.
} 
test statistic

$$
t_{s}=\frac{b_{s}}{\sqrt{V_{s s}^{R}}} .
$$

When the number of studies is large enough, the $t_{s}$ statistic approximately follows a normal distribution. ${ }^{2}$ Therefore, a $z$-test can be conducted to determine whether the meta-regression coefficient is different from zero.

To try to improve upon the CR0-type RVE, Hedges and colleagues ${ }^{2}$ and Tipton $^{9}$ examined several small-sample corrections to RVE for tests of single coefficients. Hedges and colleagues ${ }^{2}$ suggested using the adjustment matrix

$$
\mathbf{A}_{j}^{C R 1}=\sqrt{m /(m-p)} \mathbf{I}_{j}
$$

where $\mathbf{I}_{j}$ denotes a $k_{j} \times k_{j}$ identity matrix; we shall call this CR1. The CR1 adjustment matrices have the effect of inflating the CR0-type estimator by the factor $m /(m-p)$ —a rough correction for the downward bias of the estimator. Hedges and colleagues ${ }^{2}$ also proposed comparing the t-statistic to a t-distribution with $m-p$ degrees of freedom. ${ }^{2,10}$ However, simulations indicated that even with the CR1 adjustment, Type I error rates can be inflated when the number of studies is small. ${ }^{2}$

Tipton ${ }^{9}$ proposed and evaluated several further methods to improve the small-sample performance of RVE for single coefficient hypothesis tests, including the CR1 correction, a bias-reduced linearization method (CR2) proposed by McCaffrey and colleagues, ${ }^{22}$ and a jack-knife estimator (CR3). ${ }^{\dagger}$ The CR2 adjustment matrix is given by

$$
\mathbf{A}_{j}^{C R 2}=\mathbf{W}_{j}^{-1 / 2}\left[\mathbf{W}_{j}^{-1 / 2}\left(\mathbf{W}_{j}^{-1}-\mathbf{X}_{\mathbf{j}} \mathbf{M} \mathbf{X}_{j}^{\prime}\right) \mathbf{W}_{j}^{-1 / 2}\right]^{-1 / 2} \mathbf{W}_{j}^{-1 / 2}
$$

where $\mathbf{W}_{j}^{-1 / 2}$ denotes the inverse of the symmetric square root of the matrix $\mathbf{W}_{j}$. Tipton and Pustejovsky ${ }^{10}$ demonstrated that when the working model is correct, using the CR2 adjustment matrix provides an exactly unbiased estimator of Var(b). The CR3 adjustment matrix is given by

$$
\mathbf{A}_{j}^{C R 3}=\sqrt{\frac{m}{m-1}}\left(\mathbf{I}_{j}-\mathbf{X}_{\mathbf{j}} \mathbf{M} \mathbf{X}_{j}^{\prime} \mathbf{W}_{j}\right)^{-1},
$$

which is a close approximation to the jack-knife variance estimator. Along with the adjustment matrices, Tipton ${ }^{9}$ proposed using a Satterthwaite correction for degrees of freedom. The Satterthwaite degrees of freedom are calculated as

$$
v_{s}=\frac{2 \mathrm{E}\left(V_{s s}^{R}\right)^{2}}{\operatorname{Var}\left(V_{s s}^{R}\right)}
$$

where $\mathrm{E}\left(V_{s s}^{R}\right)$ and $\operatorname{Var}\left(V_{s s}^{R}\right)$ are approximated based on the working model. ${ }^{9,10}$

Tipton ${ }^{9}$ ran two simulation studies to examine these small-sample correction methods, with and without the Satterthwaite degrees of freedom, in terms of Type I error rates, power, and degrees of freedom. Results demonstrated that all the methods performed poorly, resulting in Type I error rate inflation when used without any degrees of freedom correction. However, all methods performed well when combined with the Satterthwaite correction, so long as the degrees of freedom were greater than or equal to four. The CR2 and CR3 methods combined with the Satterthwaite degrees of freedom showed better Type I error rate control than the $\mathrm{CR} 1$ method, but $\mathrm{CR} 3$ was more conservative than $\mathrm{CR} 2$.

Tipton $^{9}$ also showed that the number of studies was not the only factor that influences the Type I error rates of RVE. High leverage also influences the performance of CR0 and CR1-type RVE. The CR2 adjustment matrices and Satterthwaite degrees of freedom account for leverage, leading to tests with better-calibrated Type I error rates.

\subsection{2 | Tests of Multiple-Contrast Hypotheses}

In addition to tests of single coefficients, meta-analysts are also often interested in tests of multiple-contrast hypotheses, such as when testing for a moderating effect of a categorical variable with multiple levels or when comparing nested meta-regression specifications. Consider the null hypothesis $\mathrm{H}_{0}: \mathbf{C} \boldsymbol{\beta}=\mathbf{0}$, where $\mathbf{C}$ denotes a $q \times p$ contrast matrix and $\mathbf{0}$ denotes a $q \times 1$ vector of zeros. Here, $q$ denotes the number of constraints. To evaluate the null hypothesis, a $Q$ statistic can be calculated as

$$
Q=\mathbf{b}^{\prime} \mathbf{C}^{\prime}\left(\mathbf{C V}^{\mathbf{R}} \mathbf{C}^{\prime}\right)^{-1} \mathbf{C b} \text {. }
$$

${ }^{\dagger}$ The three adjustment matrices are generalizations of the heteroskedasticity-consistent variance estimators HC1, HC2, and HC3 proposed by MacKinnon and White. ${ }^{23,22}$ 
The $Q$ statistic approximately follows a $\chi^{2}$ distribution with $q$ degrees of freedom when the number of studies is adequately large. ${ }^{10}$ However, this approximation is poor when the number of studies is small or when the covariate matrices include points of high leverage.

Tipton and Pustejovsky ${ }^{10}$ developed small-sample adjustments for $F$-tests of multiple-contrast hypotheses. They first considered a degrees of freedom correction similar to the correction for single coefficient tests suggested by Hedges and colleagues. ${ }^{2}$ The test statistic is calculated as $F=Q / q$, where the $Q$ statistic is calculated using the CR1 adjustment matrices. The test statistic is compared to an $F$ distribution with $q$ and $m-p$ degrees of freedom. Tipton and Pustejovsky ${ }^{10}$ called this test the Naive $F$-test.

Tipton and Pustejovsky ${ }^{10}$ also considered several other strategies to better approximate the sampling distribution of the $Q$ statistic. Let $\mathbf{D}$ denote the random matrix $\left(\mathbf{C V}^{\mathbf{R}} \mathbf{C}^{\prime}\right)^{-1}$ from Equation (10), where $\mathbf{V}^{R}$ is calculated using the CR2 adjustment matrices. One strategy uses the eigen-decomposition of $\mathbf{D}$, approximating $Q$ as a sum of squares of independent random variables, each of which follows a t-distribution with degrees of freedom estimated by a Satterthwaite approximation:

$$
Q=\sum_{r=1}^{q} t_{r}^{2}
$$

where $t_{r} \sim t\left(v_{r}\right)$ and $v_{r}$ are approximated using Equation (9). Following a strategy developed in Cai and Hayes, ${ }^{24}$ the $t$-variates are then passed through a function $g()$ that normalizes their distribution, such that $g\left(t_{r}, v_{r}\right) \dot{\sim} N(0,1)$. This yields a new statistic

$$
C=\sum_{r=1}^{q} g\left(t_{r}, v_{r}\right)^{2},
$$

which is compared to a $\chi^{2}$ reference distribution with $q$ degrees of freedom. Tipton and Pustejovsky ${ }^{10}$ referred to this test as EDT, for eigen-decomposition and transformation.

Another strategy involves approximating the sampling distribution of $\mathbf{D}$ using the Wishart distribution, leading to test statistic that approximately follows Hotelling's $T^{2}$ distribution. ${ }^{\dagger}$ The $Q$ statistic follows a Hotelling's $T^{2}$ distribution with degrees of freedom $\eta$ :

$$
\frac{\eta-q+1}{\eta q} Q \sim F(q, \eta-q+1) .
$$

Tipton and Pustejovsky ${ }^{10}$ considered three different approaches for approximating $\eta$. One of these, denoted HTZ, is based on a technique used by Zhang, ${ }^{25}$ where the degrees of freedom are estimated as

$$
\eta=\frac{q(q+1)}{\sum_{s=1}^{q} \sum_{t=1}^{q} \operatorname{Var}\left(d_{s t}\right)}
$$

where $d_{s t}$ denotes the entry in row $s$ and column $t$ of $\mathbf{D}$ and $\operatorname{Var}\left(d_{s t}\right)$ is estimated using the working model. For tests of single coefficients (i.e., $q=1$ ), the HTZ test corresponds exactly to the Satterthwaite degrees of freedom correction.

Tipton and Pustejovsky ${ }^{10}$ ran a simulation study to evaluate a range of different small-sample corrections for multiplecontrast hypothesis tests, including the Naive $F$-test, two eigen-decomposition based correction methods, and three methods based on Hotelling's $T^{2}$ approximation. The simulations in Tipton and Pustejovsky ${ }^{10}$ used a design matrix that had variables with high levels of imbalance and leverage. The results demonstrated that the Naive $F$-test performed poorly, even when the number of studies was as large as 100. The eigen-decomposition based methods, including the EDT test, exhibited Type I error rate inflation under some conditions, especially when the number of studies was small (i.e., 10 or 20 studies). The three Hotelling's $T^{2}$ based methods showed better Type I error rate control, and the HTZ test resulted in Type I error rates closest to the nominal rate. On this basis, Tipton and Pustejovsky ${ }^{10}$ recommended using the HTZ test for conducting multiple-contrast hypothesis tests in general. Although it controlled Type I error rates adequately, the HTZ test had below nominal Type I error rates across many conditions, indicating that the test might be conservative.

${ }^{\dagger}$ The Hotelling's $T^{2}$ distribution is a transformation of the $F$ distribution. ${ }^{10}$ 


\section{3 | Cluster Wild Bootstrapping}

A potential alternative to the small-sample approximations that we have described is cluster wild bootstrapping. Cluster wild bootstrapping has been investigated to correct clustered heteroskedastic error terms of regression parameters estimated by ordinary least squares, in the context of primary data analysis. ${ }^{14}$ However, as noted by Tipton and Pustejovsky, ${ }^{10}$ it has not been studied in a meta-analytic framework.

Wild bootstrapping, first proposed by $\mathrm{Liu}^{26}$ based on work by $\mathrm{Wu},{ }^{27}$ involves sampling transformed residuals. The general process of conducting a wild bootstrap test is as follows: ${ }^{14,15}$

1. Fit both a null model and a full model on the original data; calculate a test statistic based on the full model.

2. Obtain the cluster-specific residual vectors from the null model.

3. For each cluster, generate an auxiliary random variable that has mean of 0 and variance of 1 ; multiply the residual vector for that cluster by the random variable.

4. Obtain new, bootstrapped outcome scores by adding the transformed residuals to the predicted values from the null model fit on the original data.

5. Re-estimate the full model with the new, bootstrapped outcome scores and calculate the test statistic.

Steps 3-5 are repeated over $R$ bootstrap replications. The p-value for the test comparing the null model to the full model is calculated as the proportion of times that the bootstrap test statistic is more extreme than the test statistic calculated from the original data. ${ }^{14,12}$

For bootstrap-based hypothesis tests, MacKinnon ${ }^{28}$ recommended imposing the null hypothesis when calculating the residuals so that the bootstrap re-sampling process more closely approximates the distribution of a test statistic when the null hypothesis is true. Furthermore, according to MacKinnon, ${ }^{28}$ imposing a null hypothesis makes the bootstrap test more reliable because nuisance parameters, which may influence the distribution of the test statistic, are more efficiently estimated. The null model contains predictors that are not being tested in single coefficient tests or multiple-contrast hypothesis tests. The full model, on the other hand, includes all the predictors of interest.

Cameron and colleagues ${ }^{14}$ extended the wild bootstrap for analyses involving clusters, proposing that the auxiliary random variable be constant within clusters. Because the weights are constant within each cluster, bootstrap-based inferences preserve the within-cluster variances and covariances of the error terms to the extent that the residuals preserve them. ${ }^{29}$ Therefore, the cluster wild bootstrap method is appropriate for regressions involving dependent errors.

The main assumption underlying wild and cluster wild bootstrapping is that the functional form of the full model is specified correctly. Like residual bootstrapping, wild and cluster wild bootstrapping involves holding $\mathbf{X}$ constant. This feature is particularly useful in cases where there are few clusters because it prevents lack of variance in the bootstrapped covariate matrix due to re-sampling. ${ }^{14}$ Unlike residual bootstrapping, cluster wild bootstrapping does not require the regression error vectors to be identically and independently distributed or the clusters to have the same sample size. ${ }^{14}$ Therefore, relative to the other bootstrap re-sampling processes, cluster wild bootstrapping may be a better procedure for conducting hypothesis tests when the number of clusters is small.

Davidson and Flachaire ${ }^{30}$ and MacKinnon ${ }^{28}$ noted that the residual terms can be transformed in some way before multiplying them by the auxiliary random variable. For non-clustered data, MacKinnon ${ }^{28}$ suggested dividing the residuals by $\left(1-h_{i}\right)^{1 / 2}$, with $h_{i}$ denoting the $i^{t h}$ diagonal element of the hat matrix. This transformation corresponds to the HC2 adjustment for heteroskedasticity-robust standard errors. ${ }^{30,31,28}$ MacKinnon ${ }^{28}$ noted that multiplying the residuals by the HC 2 correction ensures that the transformed residuals will have the correct variance if the actual errors have constant variance. If the residuals are not transformed with the $\mathrm{HC} 2$ correction, they will have variance that underestimates the true error variance. MacKinnon ${ }^{31}$ showed through simulations that multiplying the residuals by the $\mathrm{HC} 2$ or $\mathrm{HC} 3$ corrections when running wild bootstrap tests resulted in better Type I error rates compared to multiplying the residuals by the $\mathrm{HC} 1$ correction.

In models where errors have within-cluster dependence, one possible way to transform the residuals is to multiply the residuals by the CR2 adjustment matrices from the null model. The transformed residuals will then have the correct variance under the assumed working model. ${ }^{32}$ We consider this transformation in the subsequent simulations.

Formally, implementing a cluster wild bootstrap test entails the following. Let $\mathbf{X}_{0 j}$ denote a $k_{j} \times p_{0}$ matrix of covariates in the null model, $\tilde{\boldsymbol{\beta}}_{0}$ denote a $p_{0} \times 1$ vector of coefficients from the null model fit to the original dataset, and $\tilde{\boldsymbol{e}}_{j}$ denote a $k_{j} \times 1$ 
vector of residuals derived from the null model. Let $\mathbf{B}_{j}$ denote a $k_{j} \times k_{j}$ adjustment matrix derived from the null model. Finally, let $v_{j}^{*}$ denote the auxiliary random variable for cluster $j$. In each replication of cluster wild bootstrapping, new outcome scores are calculated with the residuals and the predicted values from the null model fit to the original dataset. Thus, the cluster wild bootstrap process is as follows:

$$
\mathbf{T}_{j}^{*}=\mathbf{X}_{0 j} \tilde{\boldsymbol{\beta}}_{0}+v_{j}^{*} \mathbf{B}_{j} \tilde{\boldsymbol{e}}_{j} .
$$

Here, $\mathbf{T}_{j}^{*}$ denotes the $k_{j} \times 1$ vector of bootstrapped effect sizes for cluster $j$. If the residuals are not corrected by any adjustment matrix, $\mathbf{B}_{j}$ will be an identity matrix.

When calculating the test statistics in each replication of cluster wild bootstrapping, the CR adjustment matrices can be used to estimate the standard errors. In simulation studies of ordinary least squares regression, MacKinnon ${ }^{31}$ estimated the t-statistic in each bootstrap replication with $\mathrm{HC} 1$ to $\mathrm{HC} 4$ corrected standard errors and did not find any substantial difference across the tests in terms of Type I error rates. However, the tests differed in power, with HC1 showing the highest power and HC2 to HC4 showing decreased power. By analogy, for cluster wild bootstrapping, using the CR0 or CR1 adjustment matrices might be appropriate. For single coefficient test, a t-test statistic can be estimated with the standard error calculated using the CR0 or CR1 adjustment matrices. For single coefficient tests and for multiple-contrast hypothesis test, an $F$ statistic can be calculated as $F=Q / q$, with $Q$ estimated using the CR0 or CR1 adjustment matrices. ${ }^{\dagger}$

\subsection{1 | Distribution of Auxiliary Random Variables}

Cluster wild bootstrapping involves sampling transformed residuals, ${ }^{14}$ which are multiplied by an auxiliary random variable that has a mean of 0 and variance of $1 .{ }^{33}$ Several possible distributions may be used for this auxiliary random variable. However, MacKinnon, ${ }^{33}$ and Djogbenou and colleagues ${ }^{34}$ have shown, through simulations, the superiority of the Rademacher distribution compared to any other distribution for analyses with 10 or more clusters. With the Rademacher distribution, the auxiliary random variable takes on the values of -1 or +1 with equal probability, ${ }^{30,33}$ resulting in moments

$$
\mathrm{E}\left[v_{r}^{*}\right]=0, \mathrm{E}\left[v_{r}^{* 2}\right]=1, \mathrm{E}\left[v_{r}^{* 3}\right]=0, \mathrm{E}\left[v_{r}^{* 4}\right]=1 .
$$

Using the Rademacher distribution thus ensures that the second and the fourth moments of the bootstrap residuals are close to the corresponding moments of the true errors. ${ }^{30}$

\subsection{2 | Methodological Studies on Cluster Wild Bootstrapping}

Cluster wild bootstrapping has been studied extensively in the econometric context, where past research has focused almost exclusively on linear regression models and extensions thereof (e.g., panel models with fixed effects) estimated by ordinary least squares. Cameron and colleagues ${ }^{14}$ conducted simulation studies to examine the finite sample properties of CR1- and CR3-type CRVE compared to paired, residual, and cluster wild bootstrapping. These simulations examined a slight modification of the CR1 adjustment, as implemented in Stata, which we refer to as CR1S. Compared to the CRVE methods and other bootstrapping methods for single coefficient t-tests, the cluster wild bootstrap tests provided better control of Type I error rates. Cluster wild bootstrapping outperformed the other methods even in conditions with a small number of clusters and unbalanced cluster sizes.

MacKinnon and $\mathrm{Webb}^{29}$ examined cluster wild bootstrapping compared to the CR1S-type CRVE for cases where cluster sizes were extremely unequal. Simulation results showed that t-tests based on cluster wild bootstrapping performed better than CRVE t-tests in terms of controlling Type I error rates. However, cluster wild bootstrapping led to below-nominal rejection rates in conditions where the number of treated clusters was extremely small.

Djogbenou and colleagues ${ }^{34}$ conducted simulations to examine inferences based on the CR1S-type CRVE and the cluster wild bootstrap test. The authors compared the restricted bootstrap, which involves imposing the null hypothesis, to the unrestricted bootstrap, which does not involve imposing the null. The authors also compared use of the Rademacher distribution to use of the Mammen distribution, another type of two-point distribution. ${ }^{35}$ The results demonstrated that the restricted cluster wild bootstrap test with the Rademacher weights performed the best compared to the other tests in terms of Type I error rates and power, even when the errors followed a skewed distribution.

MacKinnon ${ }^{33}$ examined CRVE and cluster wild bootstrapping in terms of confidence interval coverage. MacKinnon ${ }^{33}$ also compared several auxiliary distributions for cluster wild bootstrapping, including the Rademacher distributions, the Mammen distributions, a six-point distribution proposed by Webb, ${ }^{36}$ and several continuous distributions. Simulation results from

${ }^{\dagger}$ As long as the original test statistic and the bootstrap test statistics are calculated the same way, using the CR 1 correction instead of the CR0 correction will not affect the resulting $\mathrm{p}$-values because the CR1 correction involves multiplying the CR0 type adjustment matrix by a constant. 
MacKinnon ${ }^{33}$ showed that the bootstrap-based confidence intervals provided more accurate coverage than the intervals based on CR1S-type CRVE. Moreover, the intervals based on cluster wild bootstrapping using the Rademacher distributions had the most accurate coverage compared to those based on bootstrapping using any other weights.

The studies reviewed here showed that, for linear models estimated by ordinary least squares, the cluster wild bootstrap test maintained adequate Type I error rates even when the number of clusters was small and cluster sizes were imbalanced. Furthermore, Djogbenou and colleagues ${ }^{34}$ and MacKinnon ${ }^{33}$ provided evidence in favor of using the Rademacher two-point distributions.

Based on the results of the studies reviewed in this section, cluster wild bootstrapping may be an attractive alternative to the HTZ test for meta-analysis. However, the performance of cluster wild bootstrapping in the context of meta-analysis has not been examined. Furthermore, no study has compared the performance of cluster wild bootstrapping to that of the robust ttest based on the CR2 correction method with the Satterthwaite degrees of freedom, nor to that of the HTZ test. No study has examined whether multiplying residuals by adjustments matrices for cluster wild bootstrapping results in any difference in Type I error rates and power. Moreover, the performance of cluster wild bootstrapping for multiple-contrast hypothesis tests has not been examined.

\section{3 | EMPIRICAL APPLICATION}

To demonstrate the differences between the methods reviewed in the previous section, we re-analyzed data from a systematic review and synthesis involving dependent effect sizes. Tanner-Smith and Lipsey ${ }^{37}$ evaluated the effectiveness of brief alcohol interventions for reducing alcohol consumption and alcohol-related problems among adolescents and young adults. Our reanalysis limited the sample to individually randomized control trials with complete covariate information, and we focused on effect sizes for alcohol consumption outcomes. To illustrate differences between methods when the number of studies is limited, we analyzed a randomly selected subset of 20 studies that met these criteria. We considered a meta-regression relating effect sizes to average age of the sample and to the type of dependent variable, which was categorized as blood alcohol concentration, combined measures, frequency of heavy use, frequency of use, peak consumption, and quantity of use. The full model included both age and the dependent variable type as predictors, and we conducted hypothesis tests for the coefficient on age (a single coefficient test) and for equality across dependent variable types (a multiple-contrast test). For each hypothesis, we compared the Naive $F$-test, the EDT test, the HTZ test, and the cluster wild bootstrap test with and without multiplying the residuals by the CR2 adjustment matrices (i.e., the CWB Adjusted and the CWB tests, respectively).

We conducted the analyses using $\mathrm{R}$ Version 4.1.2. ${ }^{38}$ We used the robu() function from the robumeta package to fit the meta-regression models with a correlated effects working model. ${ }^{39}$ We used the Wald_test() function from the clubSandwich package to run small-sample corrections for tests of both single coefficient and multiple-contrast hypotheses. ${ }^{40} \mathrm{We}$ implemented the CWB and the CWB Adjusted tests using the Wald_test_cwb() function from the wildmeta package. ${ }^{20}$ For both CWB tests, we used Rademacher weights and 999 bootstrap replications. We calculated $F$-test statistics from the original full model and from each bootstrap replication using CR0-type RVE and the Naive $F$-test.

Table 1 shows the results for the single coefficient test of average sample age and the multiple-contrast test for differences in average effects by type of dependent variable. For the single coefficient test, the p-values of the EDT, HTZ, CWB, and CWB adjusted tests are nearly identical, and all are larger than the p-value of the Naive- $F$ test. However, for the multiple-contrast test, the p-values obtained from the various small-sample approximations differ, suggesting the methods could differ in Type I error rates or power. In particular, the p-values obtained from the CWB and the CWB Adjusted tests are smaller than the p-value from the HTZ test but larger than the p-values from the Naive- $F$ test and EDT test. The results shown in Tables 1 are based on only one dataset, and so we cannot draw any general conclusions about the performance of the methods. Therefore, we conducted simulation studies to systematically examine the performance of these hypothesis testing methods under a range of conditions.

\section{4 | SIMULATION 1}

We conducted two simulation studies to evaluate the performance of cluster wild bootstrapping tests and compare them to available alternatives. Our first simulation study closely followed the design of the simulations reported by Tipton and Pustejovsky, ${ }^{10}$ as our aim was to examine a method that may provide an improvement upon methods examined in that study. We evaluated the 
Table 1 Tanner-Smith and Lipsey (2015) Analysis: Hypothesis Tests for Age and Dependent Variable Type

\begin{tabular}{lrrrrrr}
\hline & \multicolumn{2}{c}{$\begin{array}{c}\text { Sample age } \\
\left(d f_{\text {num }}=1\right)\end{array}$} & \multicolumn{4}{c}{$\begin{array}{c}\text { Dependent variable type } \\
\left(d f_{\text {num }}=5\right)\end{array}$} \\
Method & $F$ & $d f_{\text {den }}$ & $p$ & $F$ & $d f_{\text {den }}$ & $p$ \\
\hline Naive-F & 1.107 & 19 & .306 & 1.534 & 19 & .226 \\
EDT & 0.845 & $\infty$ & .358 & 1.022 & $\infty$ & .403 \\
HTZ & 0.929 & 9.97 & .358 & 0.767 & 7.15 & .601 \\
CWB $^{*}$ & 1.107 & & .357 & 1.534 & & .538 \\
CWB Adjusted $^{*}$ & 1.107 & & .360 & 1.534 & & .531 \\
\hline
\end{tabular}

* The CWB and CWB Adjusted tests do not involve denominator degrees of freedom.

Naive $F$-test, the HTZ and EDT tests developed in Tipton and Pustejovsky, ${ }^{10}$ and the CWB and CWB Adjusted tests in terms of Type I error rates and power. We programmed the simulations in R Version 3.5.1 ${ }^{38}$ and executed them using the Stampede2 supercomputer provided by the Texas Advanced Computing Center. We used the tidyverse set of packages for data-munging, ${ }^{41}$ the mvtnorm package for simulating data, ${ }^{42}$ and the simhelpers and Pusto packages for organizing the code for the simulation studies. ${ }^{43,44}$

In the remainder of this section, we outline the data generation process, estimation methods, experimental design, and performance criteria used in the first simulation study, and detail the results from the study.

\section{1 | Data Generation}

This study's data generation process closely followed that of Tipton and Pustejovsky. ${ }^{10}$ Like Tipton and Pustejovsky, ${ }^{10}$ we simulated standardized mean differences (SMDs), the most common effect size measure in meta-analyses of social science intervention research. For each replication of the simulation, we generated a meta-analytic dataset comprised of $m$ studies, where study $j$ contained $k_{j}$ effect sizes. To do so, we first generated study-specific parameters and design features, including the primary study sample sizes $N_{j}$, number of effect sizes $k_{j}$, correlation between outcomes $r_{j}$, covariates $\mathbf{X}_{j}$, and vector of effect sizes $\boldsymbol{\delta}_{j}$. Given these quantities, we then generated a vector of effect size estimates and sampling variances for each study.

\section{Study-level Parameters}

We simulated total sample sizes for each primary study as

$$
N_{j} \sim \min \{20+2 \times \operatorname{Poisson}(30), 200\} .
$$

This yielded sample sizes ranging from 20 to 200, with an average of 50. This distribution roughly followed that from Tipton and Pustejovsky ${ }^{10}$ and reflected the range of study sample sizes found in published social science meta-analyses. For example, in a meta-analysis on the effects of bilingualism on executive functioning, primary study samples ranged from 12 to 343 with a median of $48 .{ }^{45}$

We simulated the number of effect sizes per study as

$$
k_{j} \sim \min \{1+\operatorname{Poisson}(4), 10\} .
$$

The number varied from 1 to 10 effect sizes per study, with an average of 5, consistent with the range seen in real meta-analyses. In a review of 64 meta-analyses published in education and psychology journals, Tipton and colleagues, ${ }^{46}$ found an average of $4.5(\mathrm{SD}=5.6)$ effect sizes per study.

When generating correlated outcome data, we assumed a constant correlation between pairs of outcomes measured within a given study, but allowed the correlation to vary from study to study. Specifically, we simulated the correlation between outcomes in study $j$ as

$$
r_{j} \sim \operatorname{Beta}(\rho v,(1-\rho) v)
$$


where $v$ controlled the variability of $r_{j}$ across studies as $\operatorname{Var}\left(r_{j}\right)=\rho(1-\rho) /(1+v)$. Across all simulation conditions, we set the value of $v$ at 50 to generate a moderate amount of variance in the correlation values across studies. ${ }^{\dagger}$

To simulate covariates, we used the same design matrix as in Tipton and Pustejovsky, ${ }^{10}$ consisting of two binary and three continuous covariates, some balanced and normal and others imbalanced or skewed. Further details about the design matrix are available in the Supplementary Materials, Section S1.1. Tipton and Pustejovsky ${ }^{10}$ noted that these types of variables are common in applied meta-analyses, with the covariates that have large imbalances or high skewness representing the worst cases. The generated data had 200 rows, with 10 rows per study, totaling 20 studies. Following the procedures of Tipton and Pustejovsky, ${ }^{10}$ in cases where there were more than 20 studies, we repeated the rows of the design matrix. For studies with fewer than 10 effect sizes, we selected the first $k_{j}$ rows from the design matrix.

Given the covariate matrix for study $j$, we generated a vector of true effect size parameters based on a correlated effects model. Let $\delta_{i j}$ denote the true SMD $i$ in study $j, \mathbf{x}_{i j}$ denote the corresponding $1 \times 5$ row vector of covariates, $\beta_{0}$ denote an intercept parameter, and $\boldsymbol{\beta}=\left(\beta_{1} \beta_{2} \cdots \beta_{5}\right)^{\prime}$ denote a $5 \times 1$ vector of meta-regression coefficients. We generated a between-study sampling error term $v_{j}$ from a normal distribution with mean zero and variance $\tau^{2}$ and then calculated the true SMDs as

$$
\delta_{i j}=\beta_{0}+\mathbf{x}_{i j} \boldsymbol{\beta}+v_{j}
$$

for $i=1, \ldots, k_{j}$ and $j=1, \ldots, m$. To examine the Type I error rates, we simulated SMDs that were unrelated to the covariates, so that $\boldsymbol{\beta}=\mathbf{0}$. To examine power, we set one entry in $\boldsymbol{\beta}$ to a non-zero value.

\section{Standardized Mean Differences}

Given the study-level parameters, we generated correlated effect size estimates for each study based on the distribution of summary statistics from primary studies. Study $j$ consisted of two equally sized groups, treatment and control, and $k_{j}$ correlated outcome variables, which we assumed to be multivariate normally distributed within each group. Let $\boldsymbol{\delta}_{j}$ denote the vector of true effect sizes for study $j$ and $\boldsymbol{\Sigma}_{j}$ denote the $k_{j} \times k_{j}$ outcome variance-covariance matrix for study $j$, where the diagonal entries of $\boldsymbol{\Sigma}_{j}$ are equal to 1 and the non-diagonal entries are equal to $r_{j}$. Let $\overline{\mathbf{y}}_{T j}$ and $\overline{\mathbf{y}}_{C j}$ denote the $k_{j} \times 1$ vectors of sample means for the treatment and control groups, respectively, and $\mathbf{S}_{j}$ denote the $k_{j} \times k_{j}$ sample variance-covariance matrix of the outcomes, pooled across the treatment and control groups. We simulated the numerator of the effect size estimates as

$$
\left(\overline{\mathbf{y}}_{T j}-\overline{\mathbf{y}}_{C j}\right) \sim N\left(\boldsymbol{\delta}_{j}, \frac{4}{N_{j}} \boldsymbol{\Sigma}_{j}\right) .
$$

Under multivariate normality, the pooled sample covariance matrix follows a multiple of a Wishart distribution with $N_{j}-2$ degrees of freedom and scale matrix equal to $\boldsymbol{\Sigma}_{j}{ }^{47}$ :

$$
\left(N_{j}-2\right) \mathbf{S}_{j} \sim \text { Wishart }\left(N_{j}-2, \boldsymbol{\Sigma}_{j}\right) .
$$

We generated the pooled standard deviations of the outcome variables in study $j$ by simulating a single Wishart matrix, extracting the diagonal elements, dividing them by $N_{j}-2$, and taking the square root. We calculated effect size estimate $i$ in study $j$ as

$$
d_{i j}=\frac{\bar{y}_{T i j}-\bar{y}_{C i j}}{s_{i j}}
$$

where $s_{i j}$ is the $i^{t h}$ diagonal element of $\mathbf{S}_{j}$. We applied Hedges's $g$ bias correction and calculated the sampling variances of the SMDs based on conventional formulas. ${ }^{48}$

\section{2 | Estimation Methods}

For computational efficiency, we used a custom-written version of the robu() function from the robumeta package to estimate meta-regressions with a correlated effects working model. For each simulated meta-analytic dataset, we fit a model including the main effects of all of the covariates (the full model), using the correlated effects working model with the default assumption of $\rho=0.8$. Based on this model, we calculated single coefficient tests for each of the five covariates. For multiple-contrast hypotheses tests, we conducted hypothesis tests of all possible combinations of 2 to 5 regression coefficients, totaling 26 different sets of coefficients. In the conditions designed to test power, we only calculated hypothesis tests for covariate sets that included a non-zero coefficient in the parameter vector $\boldsymbol{\beta}$.

\footnotetext{
${ }^{\dagger}$ Specifically, the standard deviation of $r_{j}$ is equal to 0.070 when $\rho=.5$ and to 0.056 when $\rho=.8$.
} 
Table 2 Data Generating Conditions: Simulation 1

\begin{tabular}{ll}
\hline Parameter & Values \\
\hline Number of studies $(m)$ & $10,20,40,80$ \\
Between-study heterogeneity $(\tau)$ & $0.1,0.3$ \\
Correlation between outcomes $(\rho)$ & $0.5,0.8$ \\
Regression coefficients $(\boldsymbol{\beta})$ & A. $\beta_{0}=0.3, \beta_{1}, \ldots, \beta_{5}=0$ \\
& B. $\beta_{0}=0.3, \beta_{1}=0.1$ or $0.5, \beta_{2}, \ldots, \beta_{5}=0$ \\
& C. $\beta_{0}=0.3, \beta_{2}=0.1$ or $0.5, \beta_{1}$ and $\beta_{3}, \ldots, \beta_{5}=0$ \\
& D. $\beta_{0}=0.3, \beta_{3}=0.1$ or $0.5, \beta_{1}, \beta_{2}, \beta_{4}, \beta_{5}=0$ \\
& E. $\beta_{0}=0.3, \beta_{4}=0.1$ or $0.5, \beta_{1}, \ldots, \beta_{3}$ and $\beta_{5}=0$ \\
& F. $\beta_{0}=0.3, \beta_{5}=0.1$ or $0.5, \beta_{1}, \ldots, \beta_{4}=0$ \\
\hline
\end{tabular}

For each hypothesis test on each simulated dataset, we calculated five distinct tests. We used the Wald_test() function from the clubSandwich package ${ }^{40}$ to calculate the Naive $F$, EDT, and HTZ tests. For the Naive $F$-test, we used the CR1 variance-covariance matrix. For the EDT and HTZ tests, we used the CR2 small-sample adjustment matrices.

For the bootstrap-based tests, we estimated the null model (i.e., excluding the covariate or covariates being tested) using the custom robu() function. We calculated the residuals and predicted values from the null model, along with the CR2 adjustment matrices. To examine whether transformation of the residuals influenced the calibration or power of the bootstrap tests, we computed the cluster wild bootstrap with and without multiplying the residuals by the CR2 adjustment matrices. For both tests, we used the Rademacher distribution to generate the auxiliary random variables, consistent with the recommendations of Djogbenou and colleagues ${ }^{34}$ and MacKinnon. ${ }^{33}$ We used the CR0 adjustment matrices to calculate the $F$ statistics for each bootstrap replication. We set the number of bootstrap replications to 399 , following the precedent of other simulation studies on cluster wild bootstrapping. ${ }^{14,29,34}$

\section{3 | Experimental Design}

The experimental design for this study generally followed that of Tipton and Pustejovsky, ${ }^{10}$ but with fewer conditions because of the computational demands of bootstrapping. Table 2 lists the different parameters that we examined, which included the number of studies $(m)$, between-study heterogeneity in the effect sizes $(\tau)$, within-study correlation between outcomes $(\rho)$, and the regression coefficients used to generate the effect sizes $(\boldsymbol{\beta})$. Parameters were fully crossed, for a total of $4 \times 2 \times 2 \times 11=176$ unique conditions. For each condition, we generated 2,400 datasets.

\section{Number of Studies}

In a review of meta-analyses across education and psychology journals, Tipton and colleagues ${ }^{46}$ found an average of 65.5 (SD $=65.8$ ) studies. To cover realistically small to moderate sample sizes, we set the number of independent studies $(\mathrm{m})$ to 10 , 20,40 , or $80 .^{\dagger}$ We acknowledge that meta-analyses with fewer than 10 studies do occur routinely, including as part of reviews published by the What Works Clearinghouse in education research and the Cochrane Collaboration in health sciences. However, we consider it unlikely that reviews with fewer than 10 studies would focus on moderator analyses or conduct multiple-contrast hypothesis tests.

\section{Between-Study Heterogeneity}

We set the value of $\tau$ to 0.1 or 0.3 , representing small or large heterogeneity. ${ }^{49}$ Pigott ${ }^{49}$ suggested benchmarks of $0.33 \sigma^{2}, \sigma^{2}$, and $1.33 \sigma^{2}$ for small, moderate, and large $\tau^{2}$ values for between-study heterogeneity. Given the range of sample size per study, the average $\sigma^{2}$ is approximately 0.05 ; following Pigott's benchmarks, this would imply small, moderate, and large $\tau$ values of $0.13,0.22$, and 0.26 . The $\tau$ values used in the simulation are therefore roughly in line with the small and large benchmarks. The results from Tipton ${ }^{9}$ and Tipton and Pustejovsky ${ }^{10}$ were not sensitive to the values of $I^{2}$ that the authors examined in their simulations. Therefore, we only examined a small set of $\tau$ values in our simulation studies.

These values are a subset of the number of studies evaluated by Tipton and Pustejovsky, ${ }^{10}$ excluding larger numbers of studies where all tests had similar Type 1 error rates. 


\section{Correlation between Outcomes}

We set the values for $\rho$, the average correlation between outcomes from the same study, to 0.5 or 0.8 . The robumeta package uses a default of 0.8 when estimating correlated effects meta-regression models. Thus, when the true average correlation is 0.8 , the model is close to correctly specified. We included conditions with $\rho=0.5$ in order to examine the robustness of the tests when the default assumption is incorrect, so that the working model is mildly mis-specified.

\section{Meta-Regression Coefficients}

The coefficient corresponding to a moderator in a meta-regression can be thought of as an effect size-that is, a change or difference in the outcome in standard deviation units. In a review of studies on educational interventions, Kraft ${ }^{50}$ examined 747 studies that included 1,942 effect sizes. $\mathrm{Kraft}^{50}$ reported that the median effect size estimate across all the studies was 0.1 and the $90^{\text {th }}$ percentile was 0.5 . These two values were used as regression coefficients corresponding to moderator effects when generating the meta-analytic data.

We varied the meta-regression coefficient vector across eleven unique configurations. To examine the Type I error rates, we set the intercept term $\beta_{0}=0.3$ and all remaining coefficients, $\beta_{1}$ to $\beta_{5}$, equal to 0 . To examine power, we set the coefficient corresponding to one of the five covariates equal to 0.1 or 0.5 , with the intercept equal to $\beta_{0}=0.3$ and all remaining coefficients equal to 0 .

\section{4 | Performance Criteria}

We evaluated the performance of the tests in terms of Type I error and power. Type I error rate and power both pertain to the probability that a test rejects the null at a specified $\alpha$ level. ${ }^{51}$ Letting $S$ denote the number of simulation replications and $p_{s}$ denote the p-value from simulation replication $s$, we estimated the rejection rate as

$$
r_{\alpha}=\frac{1}{S} \sum_{s=1}^{S} I\left(p_{s}<\alpha\right) .
$$

We calculated an upper confidence bound for simulation error as

$$
U_{\alpha}=\alpha+1.96 \times \sqrt{\frac{\alpha(1-\alpha)}{S}} .
$$

Following Tipton and Pustejovsky, ${ }^{10}$ we examined $\alpha$ values of $0.01,0.05$, and 0.10 , with 0.05 being the most conventional.

\section{5 | Results: Type I Error Rates}

We examined the Type I error rates for all the tests. We present results for the CWB, CWB Adjusted, EDT, and HTZ tests in the main text. The Naive $F$-test results are presented in the Supplementary Figure S1. In the main text, we focus on results for the nominal $\alpha$ level of 0.05 . Results for $\alpha$ levels of 0.01 and 0.10 are presented in Supplementary Figures S2 and S3.

The box-plots for Type I error rates show the range of Type I error rates by the number of studies $(m)$ and the number of contrasts $(q)$. Each box represents a range over the rates from specific tests and covariates and for different $\tau$ and $\rho$ values. The solid lines indicate the nominal $\alpha$ level of 0.05 and the dashed lines indicate an upper confidence bound for simulation error. Tests that have Type I error rates that fall below the simulation error bounds are considered to maintain Type I error rates adequately.

Figure 1 shows the range of Type I error rates of the CWB, CWB Adjusted, EDT, and HTZ tests. We did not include the error rates of the Naive $F$-test in these graphs because they were much higher than the rates for the other tests (Figure S1). The CWB and the CWB Adjusted tests had Type I error rates near the nominal rate across most conditions. The Type I error rates of the two CWB tests were very similar across all conditions. For single coefficient tests, Type I error rates of the CWB and CWB Adjusted tests cross the upper error bound in conditions where the number of studies was 10. Section 1.2.3 of the Supplementary materials presents results for Type I error rates for single coefficient tests disaggregated by the covariate tested, detailing the types of variables for which the CWB tests had above-nominal Type I error rates. The EDT test had close to nominal Type 1 error rates in conditions with a higher number of studies and for tests with a lower number of contrasts. However, the EDT test had high Type I error rates in conditions with few studies, especially for tests of a higher number of contrasts. Type I error rates of the HTZ test tended to be below the nominal level. For conditions with fewer studies and for tests of a higher number of contrasts, the Type I error rates of the HTZ test were far below the nominal level. 


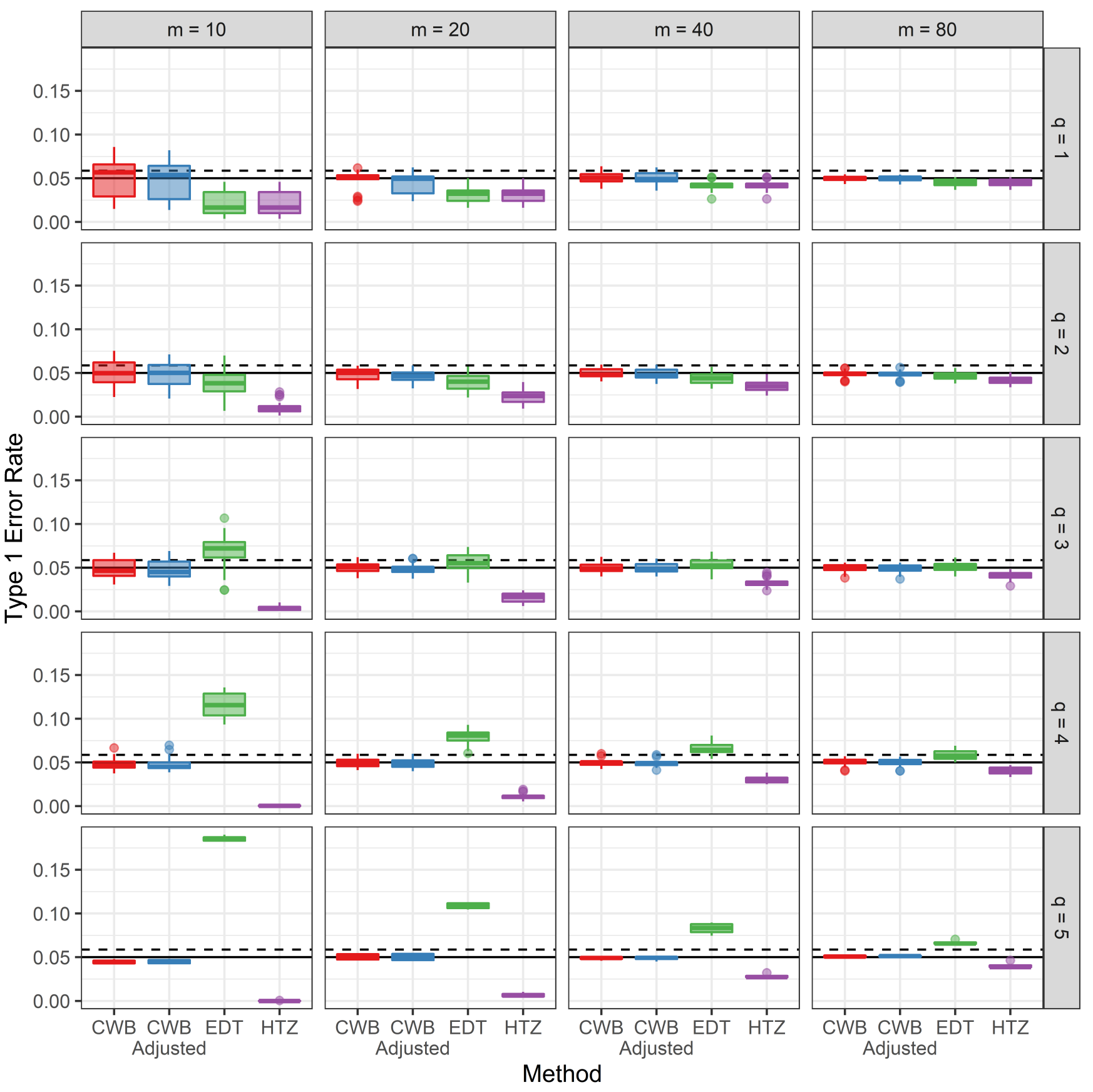

Figure 1 Study 1: Type I error rates of the CWB, CWB Adjusted, EDT, and HTZ tests by the number of studies $(m)$ and the number of contrasts $(q)$ for nominal $\alpha$ level of 0.05 . The solid lines indicate the nominal $\alpha$ level. The dashed lines indicate bounds for simulation error.

The overall pattern of results was not sensitive to the values of $\tau$ or $\rho$ used in the data-generating process. Misspecification of the working model, particularly the mis-specification of $\rho$, did not impact Type 1 error rates results. Section S1.2.4 of the Supplementary materials provides further details. 

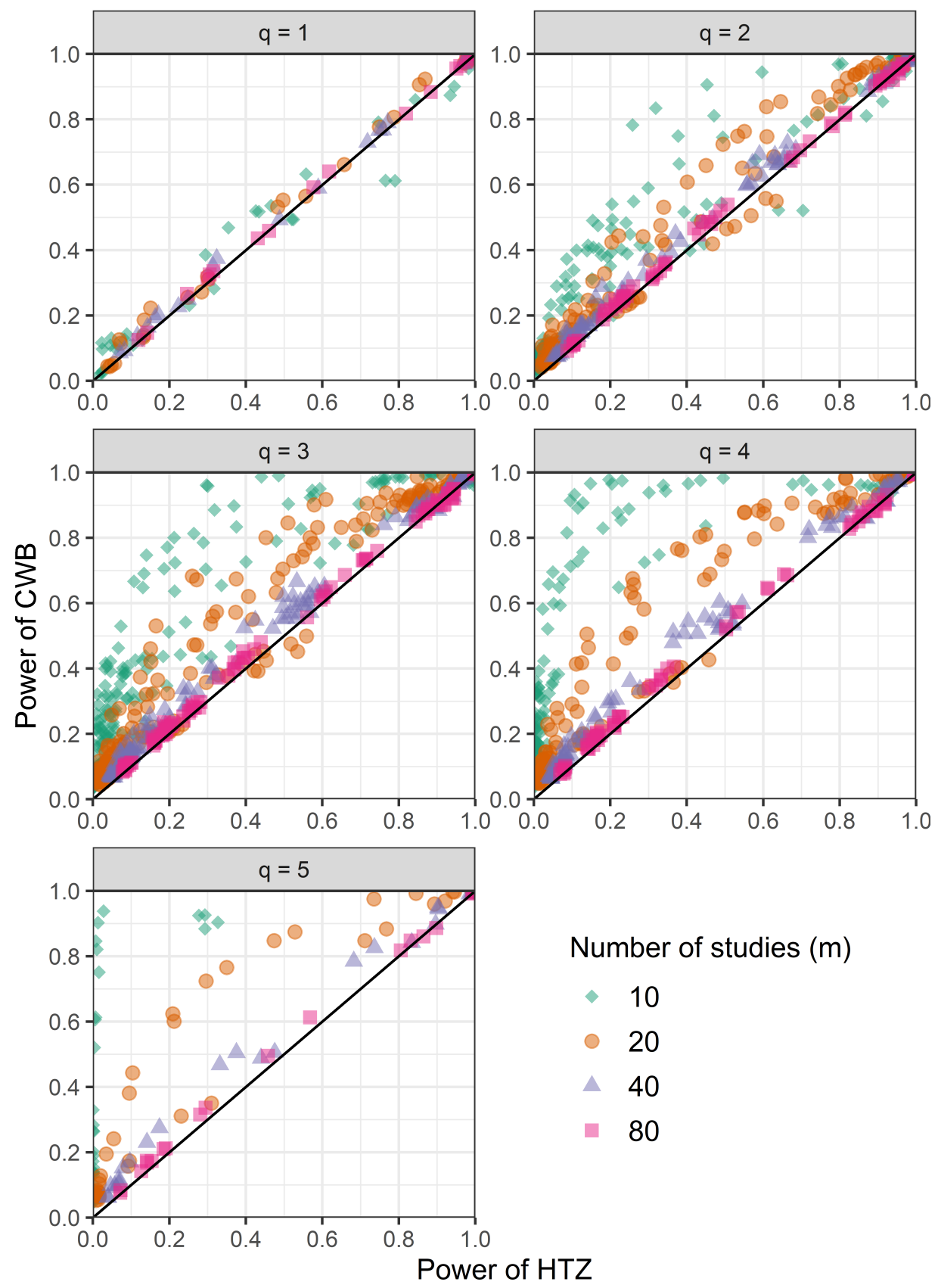

\section{Number of studies $(m)$}

$\Delta 10$

- 20

$\triangle 40$

- 80

Figure 2 Study 1: Power of the HTZ test and the CWB test by the number of contrasts $(q)$ and the the number of studies $(m)$ for nominal $\alpha$ level of 0.05 . The solid 45-degree lines correspond to equal power for the HTZ and CWB tests. Points above the solid line on the diagonal of each plot indicate that the CWB test had higher power than the HTZ test.

\section{6 | Results: Power}

For power, we only examined tests that maintained adequate Type I error rates. Therefore, we did not consider the Naive $F$-test nor the EDT test, both of which had excess Type I error rates. Furthermore, because the CWB and the CWB Adjusted tests had nearly identical power (see Supplementary Figure S9), we focused on comparing the power of the CWB test to that of the HTZ test.

Figure 2 shows the power of the HTZ and CWB tests by the number of contrasts $(q)$ and the number of studies $(m)$ for the nominal $\alpha$ level of 0.05. Results for $\alpha$ levels of 0.01 and 0.10 are presented in Supplementary Figures S10 and S11. Each 
point in the plot represents the rejection rates from specific hypothesis tests and variables, and the rates for different $\tau$ and $\rho$ values. The $\mathrm{x}$ axis contains the power of the HTZ test and the $\mathrm{y}$ axis contains the power of the CWB test. Points above the solid line on the diagonal of each plot indicate that the CWB test had higher power than the HTZ test.

Figure 2 shows that the CWB test had higher power than the HTZ test across nearly all conditions examined. In particular, for conditions with fewer studies (i.e., 10 or 20 studies) and for tests of a higher number of contrasts, the CWB test had much higher power than the HTZ test. However, in conditions with 10 or 20 studies for tests of 1 or 2 contrasts, a few of the points are slightly below the diagonal line, indicating that the HTZ test had higher power compared to the CWB test in some conditions or for some particular tests. The power differential of the HTZ test compared to the CWB test was smaller for single coefficient tests than for multiple-contrast hypotheses tests. Overall, the CWB test had higher power compared to the HTZ test.

\section{5 | SIMULATION 2}

The first simulation examined hypothesis testing procedures for a meta-regression model with multiple predictor variables, each following a different distribution. This approach replicated the design matrix studied in prior simulations on hypothesis tests for RVE. ${ }^{9,10}$ However, meta-analysts often use multiple-contrast hypothesis tests in a simpler setting, in order to test whether a single, categorical covariate explains variation in effect size magnitude, without including any further predictors-the metaanalytic analogue of one-way analysis of variance. We designed a second simulation to examine the performance of CWB and other hypothesis testing procedures when applied in this simpler context. The data-generation procedure, estimation methods, and performance criteria for this simulation were mostly similar to those for the first simulation. In what follows, we note the main differences.

\section{1 | Data Generation}

Rather than using the design matrix studied in prior work, we generated a single categorical covariate that varied either at the study-level or at the effect size-level. The covariate had $C$ levels, with $C$ equal to 3 , 4, or 5 , with every category having an equal probability of being sampled. Applied meta-analysts are likely to encounter both study-level variables (e.g., randomized versus quasi-experimental study design) and effect size-level variables (e.g., type of outcome measure). We therefore allowed the covariate to vary either at the study-level or at the effect size-level (within studies). We constrained each category to be present in at least 2 studies for the between-study covariate type and in at least 2 effect size estimates for the within-study covariate type.

\section{2 | Estimation Methods}

For Study 2, we only tested multiple-contrast hypotheses examining whether average effect sizes differed across categories. The null hypothesis was therefore that the average effects were equal across all $C$ categories.

\section{3 | Experimental Design}

Most dimensions of the experimental design for this study were identical to those from the first simulation. One major difference was the specification of the regression coefficients used to generate the true effect sizes. In all conditions, we set the intercept to 0.3 , just as in Study 1. For Type I error, we set all the $\boldsymbol{\beta}$ values except for the intercept to 0 (i.e., equal average effects in each category). For power analysis, we set the value for $\beta_{1}$, which represents the difference between the effect of the second category and that of the first category, to $0.1,0.3$, or 0.5 . All $\beta$ values expect for the intercept and $\beta_{1}$ were equal to 0 . The other major difference in design was the addition of another data generating condition, covariate type, which determined whether the covariate varied at the study-level or at the effect size-level.

Table 3 summarizes the data generating conditions varied in Study 2 . All data generating conditions were fully crossed, for a total of $4 \times 2 \times 2 \times 3 \times 2 \times 4=384$ conditions. We generated 2,400 meta-analytic datasets per condition. 
Table 3 Data Generating Conditions: Study 2

\begin{tabular}{ll}
\hline Parameter & Values \\
\hline Number of studies $(m)$ & $10,20,40,80$ \\
Between-study heterogeneity $(\tau)$ & $0.1,0.3$ \\
Correlation between outcomes $(\rho)$ & $0.5,0.8$ \\
Number of categories & $3,4,5$ \\
Covariate type & study-level, effect size-level \\
Regression coefficient $\left(\beta_{1}\right)$ & $0.0,0.1,0.3,0.5$ \\
\hline
\end{tabular}

\section{4 | Results: Type I Error Rates}

In the graphs showing the results for the Type I error rates, the rejection rates range over the rates for different $\tau$ and $\rho$ values. Because there are only 4 values per graph, we depict the results using scatter plots instead of box plots. The plots show Type I error rates by the number of studies $(m)$, the number of contrasts $(q)$, and the covariate type for nominal $\alpha$ level of 0.05 . The solid lines indicate the nominal $\alpha$ level of 0.05 and the dashed lines indicate the upper confidence bound for simulation error. We present results for $\alpha$ levels of 0.01 and 0.10 in the Supplementary Figures S13 and S14.

Figure 3 shows the range of Type I error rates of the CWB, CWB Adjusted, EDT, and HTZ tests. Overall, the pattern of results was similar to that of Study 1. The CWB and the CWB Adjusted tests maintained Type I error rates very close to the nominal $\alpha$ level. The rates exceeded the nominal level slightly in some conditions but were still within the Monte Carlo simulation error bound across most conditions. The EDT test had high Type 1 error rates for conditions with a small number of studies and for tests with a higher number of contrasts. The HTZ test had Type I error rates below the nominal rate, especially for conditions with lower numbers of primary studies and for tests involving a higher number of contrasts.

Just as in Study 1, the overall patterns of results was not sensitive to the values of $\tau$ or $\rho$. Mis-specification of the value for $\rho$ in the working model did not impact Type 1 error rates of the CWB or CWB Adjusted tests. Section S2.1.3 of the Supplementary materials provides further details.

\section{5 | Results: Power}

For consideration of power, we focused on the CWB test and the HTZ test for reasons similar to those for Study 1 (see Supplementary Figure S17). Figure 4 shows the power of the CWB test compared to that of the HTZ test by the number of contrasts $(q)$ and the number of studies $(m)$ for the study-level and effect size-level covariate types, all for the nominal $\alpha$ level of 0.05 . Results for $\alpha$ levels of 0.01 and 0.10 are presented in Supplementary Figures S18 and S19. The CWB test had higher power than the HTZ test across most conditions. In particular, for conditions with smaller number of studies (i.e., 10 or 20 studies) and for tests of higher number of contrasts, the CWB test had much higher power than the HTZ test. The overall results do not differ by covariate type, i.e., whether a covariate is study-level or effect size-level.

\section{6 | DISCUSSION}

In the form originally proposed by Hedges and colleagues, ${ }^{2}$ RVE can result in inflated Type I error rates when the number of the studies is small or when the covariates are imbalanced or have high leverage points. Previously developed small-sample corrections for hypothesis tests provide improvements over the original methods, but still have inflated Type I error rates (the EDT test) or below-nominal Type I error rates (the HTZ test). Further, results from Tipton and Pustejovsky ${ }^{10}$ suggested that the HTZ test may have low power, especially for multiple-contrast hypothesis tests. In the present study, we examined an alternative approach, based on a cluster wild bootstrapping procedure, that improves upon the existing methods. Under the conditions examined in two simulation studies, we found that tests based on cluster wild bootstrapping maintained Type I error rates near the nominal $\alpha$ level even in meta-analyses with as few as 10 studies. Moreover, CWB tests had equal or higher power than the HTZ test (the only alternative that adequately controlled Type I error rates). The power advantage of CWB was especially high for tests of a larger number of contrasts, and was apparent even in conditions with 80 studies. Based on these findings, we 

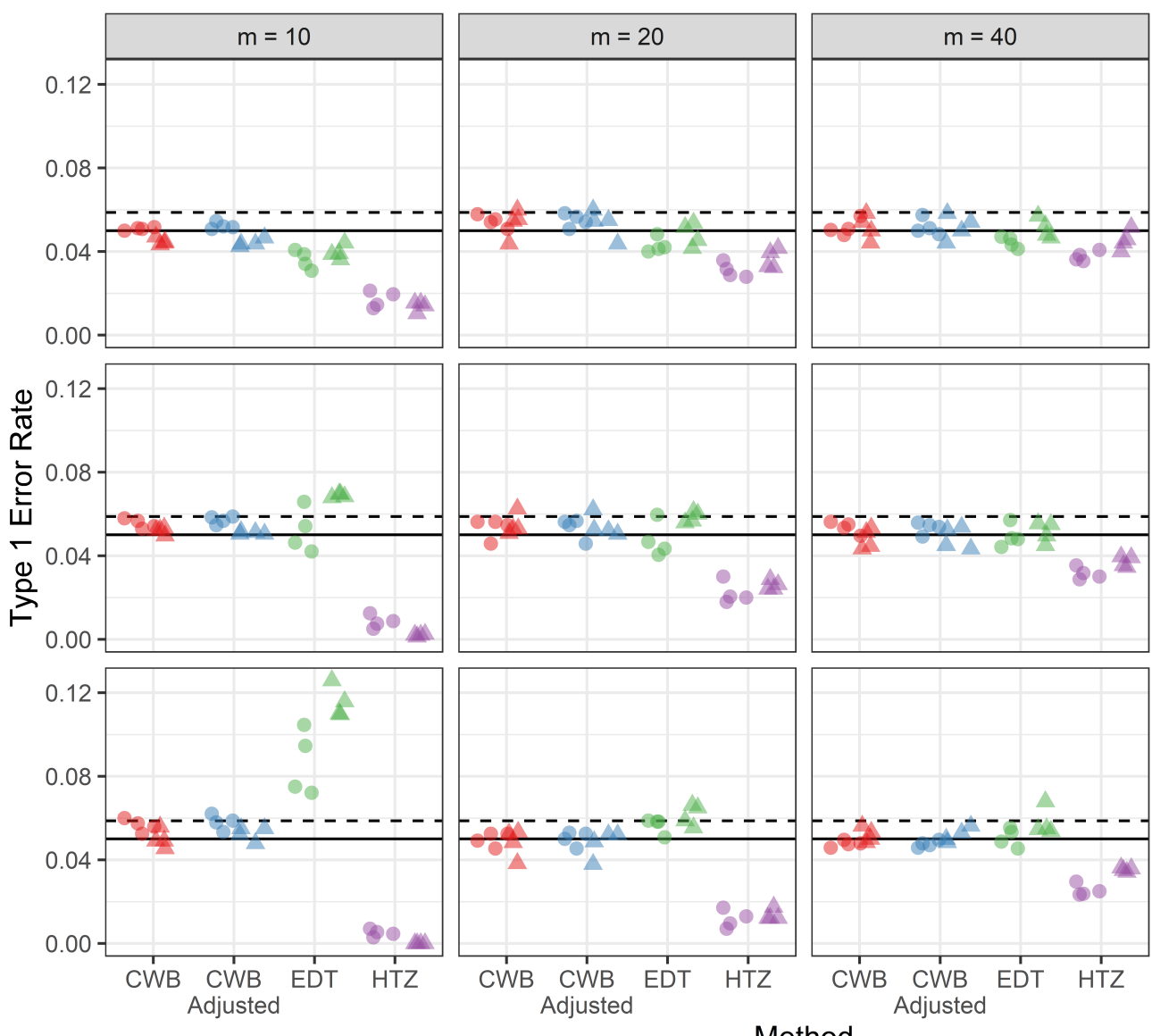

Method
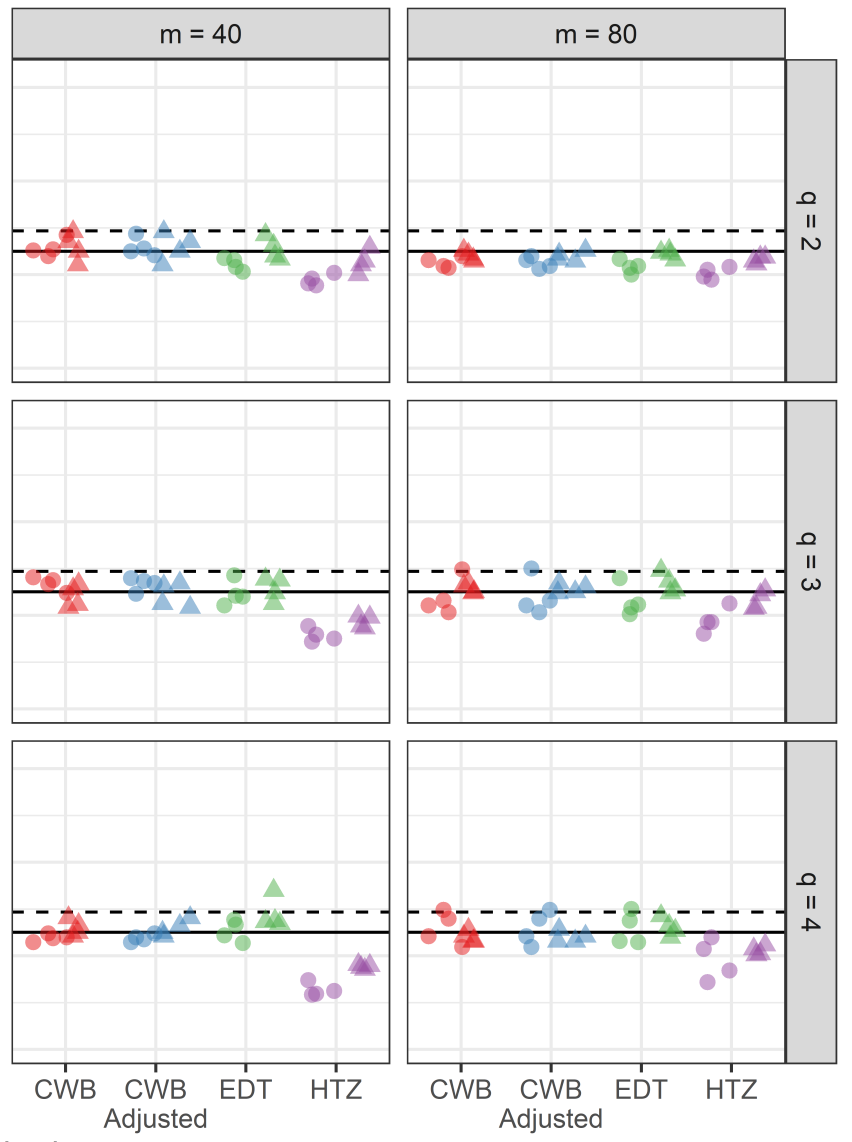

- CWB $\odot$ CWB Adjusted $\odot$ EDT $\odot \mathrm{HTZ}$

- Effect size-level $\Delta$ Study-level

Figure 3 Study 2: Type I error rates of the CWB, CWB Adjusted, EDT, and HTZ tests by the number of studies $(m)$, the number of contrasts $(q)$, and the covariate type for nominal $\alpha$ level of 0.05 . The solid lines indicate the nominal $\alpha$ level. The dashed lines indicate bounds for simulation error.

recommend using CWB rather than HTZ for tests of multiple-contrast hypotheses in meta-analyses conducted using RVE. Tests of single-contrast hypotheses (i.e., t-tests) can be conducted using either the CWB or HTZ test because both methods have very similar power.

Unlike the previously proposed small-sample corrections, the CWB test does not involve explicit small-sample corrections to the test statistic or reference distribution. Even so, the computational technique of re-sampling residuals and using the bootstrap distribution to calculate p-values resulted in better performance compared to the HTZ and EDT tests. Currently, there are scant theoretical explanations of why the CWB test out-performs alternative small-sample corrections. Liu, ${ }^{26}$ Mammen, ${ }^{35}$ and Djogbenou and colleagues ${ }^{34}$ provide theorems regarding the asymptotic validity and asymptotic refinement of the CWB test, but these do not entirely explain its remarkably accurate calibration, even when the number of studies is small. The small-sample calibration of CWB might stem in part from using the null model in the bootstrapping process, as suggested by MacKinnon, ${ }^{12}$ while also using a Wald-type test statistic (i.e., a $Q$ statistic) to measure deviations from the null. Its performance might also be due in part to re-estimation of the entire model—including the variance components and the weights used in estimating the meta-regression coefficients - with every bootstrap replication. Doing so captures the additional variability in the test statistic that arises from estimation of these components of the model. In contrast, the EDT and HTZ tests are derived by treating the variance components and weights as fixed. In future research, it would be useful to examine the necessity of re-estimating the variance components with every bootstrap replication. If it is possible to get similar performance while holding the variance 


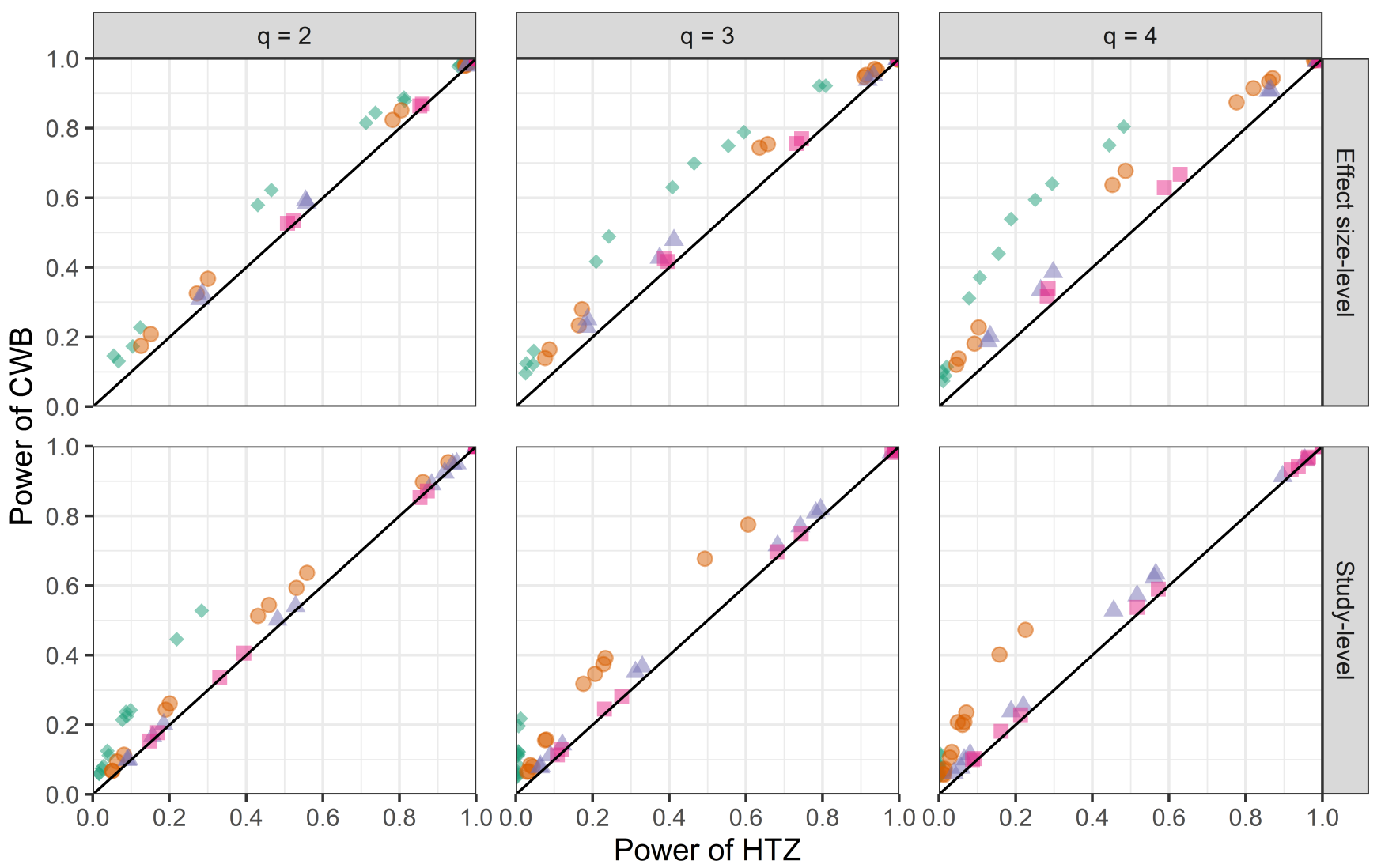

Number of studies $(\mathrm{m}) \quad 10 \bigcirc 20 \triangle 40 \quad 80$

Figure 4 Study 2: Power of the HTZ test and the CWB test by the number of contrasts $(q)$, the covariate type, and the number of studies $(m)$ for nominal $\alpha$ level of 0.05 . The solid 45-degree lines correspond to equal power for the HTZ and CWB tests. Points above the solid line on the diagonal of each plot indicate that the CWB test had higher power than the HTZ test.

components fixed (i.e., using the estimates from the null model), computation time and complexity could be improved using techniques described by MacKinnon. ${ }^{52}$

We considered two variants of cluster wild bootstrapping, one based on raw residuals (CWB) and one with a smallsample bias adjustment to the residuals used in the bootstrap process (CWB Adjusted). Across both simulations, the variants had very similar Type I error rates and power. Such similarity runs counter to previous findings from MacKinnon, ${ }^{31}$ who looked at non-clustered wild bootstrap tests for ordinary least squares regression. In the cluster wild bootstrap context, the raw residuals underestimate the error variance even when the working model is correct. ${ }^{32}$ Multiplying the residuals by the CR2 adjustment matrices should correct the under-estimation of the error variance-exactly so, when the working model is correct, and approximately so, even if the working model is incorrect. ${ }^{32}$ Our simulation studies only examined mild deviations from the working model (i.e, specification of the $\rho$ value to 0.5 instead of the within-study correlation value of 0.8 as used by robumeta). Perhaps more severe deviations from the working model—for example, using a hierarchical effects working model when the actual data structure is correlated effects-would result in more consequential differences between the performances of the CWB and the CWB Adjusted tests. Nonetheless, because the differences between the tests are very small, we see it as reasonable to use CWB without adjustment because it is conceptually and algorithmically simpler than CWB Adjusted.

In our simulations, we implemented CWB using the Rademacher distribution for the auxiliary random variable and with 399 bootstrap replications. We did not consider other distributions for the auxiliary random variable. However, we can recommend use of the Rademacher weights for meta-analyses with as few as 10 studies, based on findings from previous simulations 
by Davidson and Flachaire ${ }^{30}$ and MacKinnon. ${ }^{33}$ We used 399 bootstrap replications in the simulations purely due to computational feasibility. In practice, we recommend using a higher number of bootstrap replications, such as 1999 replications for an $\alpha=.05$-level test. Using more replications will improve power, ${ }^{53}$ at the cost only of increased computation time.

\section{1 | Limitations and Future Directions}

We examined the performance of CWB in two large simulation studies with different design matrices, in an effort to cover conditions similar to what meta-analysts encounter in practice. Still, findings from these simulations have several limitations that are important to recognize. Due to the computationally intensive nature of bootstrapping, we only examined a limited set of simulation conditions. In particular, we did not vary the distribution of primary study sample sizes, the distribution of the number of effect sizes per study, or the joint distribution of the covariates in meta-regressions involving multiple predictor variables. More variable distributions of these characteristics might negatively impact the performance of both the previously proposed tests and CWB methods. ${ }^{29}$ However, we would speculate that CWB would be relatively less affected than the EDT or HTZ tests by an extreme distribution of study characteristics. Such extreme distributions lead to conditions where the small-sample corrections involved in the EDT and HTZ tests are more influential, and thus more prone to deviation from nominal Type I error rates. We found that the CWB test works even without incorporating these small-sample corrections, which suggests that its performance might be less influenced by the distribution of study characteristics. Still, further investigation is warranted regarding the robustness of CWB tests in meta-analyses with very unequal primary study sample sizes, highly variable distributions for the number of effect sizes in each study, or multicollinear predictors.

Moreover, our simulations focused only on one effect size metric, the standardized mean difference. Hedges ${ }^{54}$ has described the fundamental unity of meta-analytic methods, arguing that meta-analytic methodology works similarly for many different effect size metrics, so long as effect size estimators are approximately unbiased and normally distributed with accurately estimated sampling variances. On the basis of this principle, we would expect that the findings from our simulations should generalize to analyses conducted with other effect size metrics, such as log-odds ratios and correlations. Future studies should examine whether this theory holds in practice—particularly for contexts with small primary study sample sizes or less commonly considered effect size metrics.

We examined only two different values for each of $\tau$ and $\rho$. However, Tipton and Pustejovsky ${ }^{10}$ and our study found that the results for small-sample corrections and CWB are not sensitive to values of $\tau$ and $\rho$ (see Supplementary materials sections S1.2.4 and S2.1.3). Furthermore, Tipton and Pustejovsky ${ }^{10}$ and our paper used methods-of-moments estimator of $\tau$. We can expect different estimators such as maximum likelihood or restricted maximum likelihood to produce fairly similar values of $\tau$. Because Type I error rates and power results of the small-sample correction methods and CWB are not sensitive to differing values of $\tau$, we would anticipate that CWB should perform similarly when variance components are estimated using likelihood-based methods.

Following the precedents of previous simulations, ${ }^{9,10}$ our simulations were limited to an examination of tests based on a correlated effects working model, under data-generating models that also had a correlated effects structure. Pustejovsky and Tipton ${ }^{21}$ introduced further types of working models that combine correlated and hierarchical effects structures, demonstrating that these new working models can lead to improvements in precision compared to the correlated effects model. Future studies should examine the performance of the CWB and HTZ tests for different kinds of dependent data structures, as well as working models that are more discrepant from the true data-generating process.

Our simulations were also limited to methods based on RVE. Meta-analysts have also explored multi-level meta-analytic models as a technique for handling dependent effect sizes, particularly when the dependence structure is hierarchical. Smallsample corrections for multi-level meta-analysis models, such as Satterthwaite or Kenward-Roger corrections, ${ }^{55}$ have yet to be investigated in meta-analyses, but might have performance characteristics similar to cluster wild bootstrap tests for multi-level meta-analytic models.

In addition to the future directions that we have noted, it would be particularly helpful to examine the implementation of multiple-contrast hypothesis tests, methods to handle dependence, and small-sample correction methods for meta-analytic data containing missing values in the moderators. Applied meta-analysts are likely to encounter missing data in the moderators because some primary studies may not provide all the information needed to code predictor variables. ${ }^{56}$ Schauer and colleagues ${ }^{57}$ are currently developing and testing multiple imputation methods for meta-analyses with dependent data. Once these techniques are more established with typical meta-analytic methods, future studies should extend them to integrate with cluster wild bootstrapping. 
A final limitation is that our investigation has focused only on techniques for testing a specific type of hypothesis, which can be specified as a set of linear constraints on meta-regression coefficients. There remains a need to develop methods for other classes of tests, such as pairwise comparison tests analogous to Tukey's honest significant differences or tests for selective reporting of study results, that work with dependent effect sizes. Bootstrapping methods have recently been explored for refining tests for selective reporting. ${ }^{58,59}$ Cluster wild bootstrapping, in particular, may prove to be useful in extending existing tests for selective reporting ${ }^{60}$ and developing other inferential techniques that can accommodate dependent effect sizes.

\section{2 | Software Implementation}

To ease the implementation of the CWB test for applied researchers, we created an R package called wildmeta, ${ }^{20}$ which is available on The Comprehensive R Archive Network (CRAN). The package includes a vignette that explains the algorithm underlying the CWB test and provides a thorough example on how to implement the CWB test for models fit with the robumeta ${ }^{39}$ and metafor ${ }^{61}$ packages.

\section{Highlights}

- Meta-analytic data often include multiple dependent effect sizes per study. A common method to handle dependence, robust variance estimation (RVE), leads to excessive false positive results when the number of studies in a meta-analysis is small. Small-sample improvements of RVE have been proposed but they have low power, especially for hypothesis tests involving more than one coefficient.

- A method known as cluster wild bootstrapping maintains adequate rates of false positive results while providing more power compared to existing small-sample corrections, especially for hypothesis tests involving multiple coefficients.

- Small samples and dependent effects are common in social science applied meta-analyses. Therefore, it is important to examine techniques to handle dependence when the number of studies is small. We evaluated a method that improves upon extant corrections by adequately controlling false positive results while providing greater power to detect true variation when it exists.

\section{Data Availability Statement}

Replication materials including code to run the simulation studies, numerical results from the studies, and code to analyze the results can be found at https://github.com/meghapsimatrix/CWB_analyses.

\section{Conflict of interest}

The authors declare no potential conflicts of interest.

\section{References}

1. Becker BJ. Multivariate meta-analysis. In: Elsevier. 2000 (pp. 499-525).

2. Hedges LV, Tipton E, Johnson MC. Robust variance estimation in meta-regression with dependent effect size estimates. Research Synthesis Methods 2010; 1(1): 39-65. doi: 10.1002/jrsm.5

3. Raudenbush SW, Becker BJ, Kalaian H. Modeling multivariate effect sizes.. Psychological Bulletin 1988; 103(1): 111.

4. Sera F, Armstrong B, Blangiardo M, Gasparrini A. An Extended Mixed-Effects Framework for Meta-Analysis. Statistics in Medicine 2019; 38(29): 5429-5444. doi: 10.1002/sim.8362

5. Wei Y, Higgins JP. Estimating within-study covariances in multivariate meta-analysis with multiple outcomes. Statistics in Medicine 2013; 32(7): 1191-1205. doi: 10.1002/sim.5679 
6. Riley RD, Thompson JR, Abrams KR. An alternative model for bivariate random-effects meta-analysis when the withinstudy correlations are unknown. Biostatistics 2008; 9(1): 172-186. doi: 10.1093/biostatistics/kxm023

7. Chen Y, Hong C, Riley RD. An alternative pseudolikelihood method for multivariate random-effects meta-Analysis. Statistics in Medicine 2015; 34(3): 361-380. doi: 10.1002/sim.6350

8. Hong C, D. Riley R, Chen Y. An Improved Method for Bivariate Meta-Analysis When within-Study Correlations Are Unknown. Research Synthesis Methods 2018; 9(1): 73-88. doi: 10.1002/jrsm.1274

9. Tipton E. Small sample adjustments for robust variance estimation with meta-regression.. Psychological Methods 2015; 20(3): 375-393. doi: 10.1037/met0000011

10. Tipton E, Pustejovsky JE. Small-sample adjustments for tests of moderators and model fit using robust variance estimation in meta-regression. Journal of Educational and Behavioral Statistics 2015; 40(6): 604-634. doi: 10.3102/1076998615606099

11. Boos DD, others . Introduction to the bootstrap world. Statistical Science 2003; 18(2): $168-174$.

12. MacKinnon JG. Bootstrap hypothesis testing. Handbook of Computational Econometrics 2009; $183: 213$.

13. Freedman DA. Bootstrapping regression models. The Annals of Statistics 1981; 9(6): 1218-1228.

14. Cameron AC, Gelbach JB, Miller DL. Bootstrap-based improvements for inference with clustered errors. The Review of Economics and Statistics 2008: 47.

15. MacKinnon JG, Webb MD. The wild bootstrap for few (treated) clusters. The Econometrics Journal 2018; 21 (2): $114-135$. doi: $10.1111 /$ ectj. 12107

16. McEwan PJ. Improving learning in primary schools of developing countries: A meta-analysis of randomized experiments. Review of Educational Research 2015; 85(3): 353-394. doi: 10.3102/0034654314553127

17. Gallet CA, Doucouliagos H. The income elasticity of air travel: A meta-analysis. Annals of Tourism Research 2014; 49: 141-155. doi: 10.1016/j.annals.2014.09.006

18. Oczkowski E, Doucouliagos H. Wine prices and Quality Ratings: A meta-regression analysis. American Journal of Agricultural Economics 2015; 97(1): 103-121. doi: 10.1093/ajae/aau057

19. Ola O, Menapace L. A meta-analysis understanding smallholder entry into high-value markets. World Development 2020; 135: 105079 .

20. Joshi M, Pustejovsky J. wildmeta: Cluster Wild Bootstrapping for Meta-Analysis. 2022. R package version 0.1.0.

21. Pustejovsky JE, Tipton E. Meta-analysis with robust variance estimation: Expanding the range of working models. Prevention Science 2021. doi: 10.1007/s11121-021-01246-3

22. McCaffrey DF, Bell RM, Botts CH. Generalizations of biased reduced linearization. In: No. 1994. ; $2001: 673$.

23. MacKinnon JG, White H. Some heteroskedasticity-consistent covariance matrix estimators with improved finite sample properties. Journal of Econometrics 1985; 29(3): 305-325.

24. Cai L, Hayes AF. A new test of linear hypotheses in OLS regression Under heteroscedasticity of unknown form. Journal of Educational and Behavioral Statistics 2008; 33(1): 21-40. doi: 10.3102/1076998607302628

25. Zhang JT. An approximate Hotelling T2-test for heteroscedastic one-way MANOVA. Open Journal of Statistics 2012; 2(1): $1-11$.

26. Liu RY. Bootstrap procedures under some non-I.I.D. models. The Annals of Statistics 1988; 16(4): 1696-1708.

27. Wu CFJ. Jackknife, bootstrap and other resampling methods in regression analysis. the Annals of Statistics 1986; 14(4): 1261-1295. 
28. MacKinnon JG. Bootstrap methods in econometrics. Economic Record 2006; 82: S2-S18.

29. MacKinnon JG, Webb MD. Wild bootstrap inference for wildly different cluster sizes. Journal of Applied Econometrics 2017; 32(2): 233-254. doi: 10.1002/jae.2508

30. Davidson R, Flachaire E. The wild bootstrap, tamed at last. Journal of Econometrics 2008; 146(1): 162-169. doi: 10.1016/j.jeconom.2008.08.003

31. MacKinnon JG. Thirty years of heteroskedasticity-robust inference. In: Springer. 2013 (pp. 437-461).

32. Pustejovsky JE, Tipton E. Small-sample methods for cluster-robust variance estimation and hypothesis testing in fixed effects models. Journal of Business \& Economic Statistics 2018; 36(4): 672-683. doi: 10.1080/07350015.2016.1247004

33. MacKinnon JG. Wild cluster bootstrap confidence intervals. L'Actualité Économique 2015; 91(1-2): 11-33.

34. Djogbenou AA, MacKinnon JG, Nielsen MØ. Asymptotic theory and wild bootstrap inference with clustered errors. Journal of Econometrics 2019; 212(2): 393-412.

35. Mammen E. Bootstrap and wild bootstrap for high dimensional linear models. The Annals of Statistics 1993: 255-285.

36. Webb MD. Reworking wild bootstrap based inference for clustered errors. tech. rep., Queen's Economics Department Working Paper; 2013.

37. Tanner-Smith EE, Lipsey MW. Brief alcohol interventions for adolescents and young adults: A systematic review and meta-analysis. Journal of substance abuse treatment 2015; 51: 1-18.

38. R Core Team . R: A Language and Environment for Statistical Computing. R Foundation for Statistical Computing; Vienna, Austria: 2022.

39. Fisher Z, Tipton E, Zhipeng H. robumeta: Robust Variance Meta-Regression. 2017. R package version 2.0.

40. Pustejovsky JE. clubSandwich: Cluster-Robust (Sandwich) Variance Estimators with Small-Sample Corrections. 2020. R package version 0.4.2.

41. Wickham H, Averick M, Bryan J, et al. Welcome to the tidyverse. Journal of Open Source Software 2019; 4(43): 1686. doi: 10.21105/joss.01686

42. Genz A, Bretz F, Miwa T, et al. mvtnorm: Multivariate Normal and t Distributions. 2020. R package version 1.1-0.

43. Joshi M, Pustejovsky JE. simhelpers: Helper Functions for Simulation Studies. 2020. R package version 0.1.0.

44. Pustejovsky JE. Pusto: Pusto's miscellaneous data analysis and simulation tools. 2018. R package version 0.3.0.

45. Lehtonen M, Soveri A, Laine A, Järvenpää J, De Bruin A, Antfolk J. Is bilingualism associated with enhanced executive functioning in adults? A meta-analytic review.. Psychological bulletin 2018; 144(4): 394.

46. Tipton E, Pustejovsky JE, Ahmadi H. Current practices in meta-regression in psychology, education, and medicine. Research Synthesis Methods 2019; 10(2): 180-194.

47. Anderson T, Girshick M. Some extensions of the Wishart distribution. The Annals of Mathematical Statistics 1944; 15(4): 345-357.

48. Borenstein M, Hedges LV. Effect sizes for Meta-Analysis. The Handbook of Research Synthesis and Meta-Analysis 2019: 207-243.

49. Pigott TD. Advances in Meta-Analysis. Springer Science \& Business Media . 2012.

50. Kraft MA. Interpreting effect sizes of education interventions. Educational Researcher 2020; 49(4): 241-253.

51. Morris TP, White IR, Crowther MJ. Using simulation studies to evaluate statistical methods. Statistics in medicine 2019; 38(11): 2074-2102. 
52. MacKinnon JG. Fast cluster bootstrap methods for linear regression models. tech. rep., Queen's Economics Department Working Paper; 2021.

53. Davidson R, MacKinnon JG. Bootstrap tests: How many bootstraps?. Econometric Reviews 2000; 19(1): 55-68.

54. Hedges LV. Statistical considerations. The Handbook of Research Synthesis and Meta-Analysis 2009: 37-47.

55. Kenward MG, Roger JH. An improved approximation to the precision of fixed effects from restricted maximum likelihood. Computational Statistics \& Data Analysis 2009; 53(7): 2583-2595.

56. Pigott TD. Handling missing data. The Handbook of Research Synthesis and Meta-Analysis 2019; 3: 367-381.

57. Schauer JM, Diaz K, Pigott TD, Lee J. Exploratory Analyses for Missing Data in Meta-Analyses and Meta-Regression: A Tutorial. Alcohol and Alcoholism 2021.

58. Duan R, Piao J, Marks-Anglin A, et al. Testing for publication bias in meta-analysis under Copas selection model. 2020.

59. Lin L. Hybrid test for publication bias in meta-analysis. Statistical Methods in Medical Research 2020; 29(10): 2881-2899.

60. Rodgers MA, Pustejovsky JE. Evaluating Meta-Analytic Methods to Detect Selective Reporting in the Presence of Dependent Effect Sizes. Psychological Methods 2021; 26(2): 141-160.

61. Viechtbauer W. Conducting meta-analyses in R with the metafor package. Journal of statistical software 2010; 36(3): 1-48. 